\title{
GEOLOGY AND GEOCHEMISTRY OF THE EARLY PROTEROZOIC KORTEJÄRVI AND LAIVAJOKI CARBONATITES, CENTRAL FENNOSCANDIAN SHIELD, FINLAND
}

\author{
JUHA NYKÄNEN, KAUKO LAAJOKI and JUHA KARHU
}

JUHA NYKÄNEN, KAUKO LAAJOKI and JUHA KARHU, 1997: Geology and Geochemistry of the Early Proterozoic Kortejärvi and Laivajoki Carbonatites, Central Fennoscandian Shield, Finland. Bull.Geol. Soc. Finland 69, Part 1-2, 5-30.

This paper provides for the first time extensive petrological, mineralogical and geochemical data on the early Proterozoic Kortejärvi and Laivajoki carbonatites, northern Finland, which form metamorphosed and highly strained bodies 2 and $4 \mathrm{~km}$ long within a Svecokarelian shear zone in central Fennoscandian Shield. They are not exposed, but have been penetrated by a couple of deep drill holes.

In terms of modal mineralogy, both intrusions contain calcite carbonatite and dolomite-calcite carbonatite as their main rock types, but Kortejärvi also contains dolomite carbonatite and calcite-dolomite carbonatite, some glimmerite and olivine-magnetite rock and Laivajärvi tremolite-calcite carbonatite, tremolite-dolomite carbonatite, serpentine-talc-dolomite rock and glimmerite. The main country rock is an amphibolite which is not fenitized. No alkaline rocks have been detected in these intrusions.

Calcite is most common mineral in both occurrences. Other carbonate minerals include dolomite with minor ankerite and occassional siderite. In addition to low-Ti phlogopite, tetraferriphlogopite is also encountered. Fresh olivine is rare, and its alteration products include titaniferous clinohumite. The amphiboles are mainly calcic amphiboles, including actinolite, tremolite and edenite. The only sodic-calcic amphibole is accessory richterite. Other essential minerals are Ti-poor magnetite with ilmenite exsolutions, fluorapatite (3.95-4.89 wt. \% F), monazite, and allanite-(Ce).

Geochemically, the Kortejärvi rocks are mostly magnesiocarbonatites, whereas those of Laivajärvi, due to their higher magnetite content, are ferrocarbonatites. Of the trace elements, $\mathrm{Nb}$ is much lower $(8-30 \mathrm{ppm})$ in proper carbonatites than the average for carbonatites and $U$ and Th $(\leq 0.9 \mathrm{ppm}$ and $\leq 2.4 \mathrm{pm}$, respectively) lower than average. Sr is typical, but not high (1830$3480 \mathrm{ppm})$, and $\mathrm{Ba}$ is rather low (27-348 ppm). The REEs are hosted by allanite and monazite and their concentrations in the proper carbonatites are relatively low $(\operatorname{REE}(\mathrm{Y})$ tot $737-1452 \mathrm{ppm})$, but the LREE/HREE ratios are markedly high (La/Lu 244-1092). 
The mean $\delta^{13} \mathrm{C}$ values of dolomite and calcite for the Laivajoki samples are $-4.3 \pm 0.1 \%$ ( $\pm 1 \mathrm{sd}, \mathrm{PDB})$ and $-4.5 \pm 0.6 \%$, respectively, and for the Kortejärvi samples $-3.9 \pm 0.2 \%$ o and $-4.2 \pm 0.2 \%$, respectively. The mean $\delta^{18} \mathrm{O}$ values of dolomite and calcite for the Laivajoki samples are $6.5 \pm 0.5 \%$ o $( \pm 1 \mathrm{sd}$, SMOW) and $7.0 \pm 0.8 \%$, respectively, and for the Kortejärvi intrusion $6.8 \pm 0.1 \%$ o and $8.4 \pm 1.0 \%$ o, respectively. These isotopic characteristics are similar to the analytical results reported from other carbonatite complexes and are in the range expected for carbonate melts in equilibrium with mantle minerals.

The Kortejärvi and Laivajärvi intrusions represent slightly metasomatized early - middle stage carbonatites which were metamorphosed in amphibolite facies and sheared during the Svecokarelian orogeny about $1880-1800 \mathrm{Ma}$ ago.

Keywords: carbonatites, mineralogy, calcite, dolomite, ankerite, siderite, glimmerite, phlogopite, amphibole group, magnetite, ilmenite, olivine, apatite, monazite, allanite, geochemistry, trace elements, rare earths, stable isotopes, Proterozoic, Kortejärvi, Laivajoki, Petäikkö-Suvantovaara, Finland

Juha Nykänen and Kauko Laajoki, Department of Geology, University of Oulu, Linnanmaa, 90570 Oulu, Finland.

Juha Karhu, Geological Survey of Finland, Betonimiehenk. 4, 00250 Espoo, Finland.

\section{INTRODUCTION}

Carbonatites are relatively rare igneous rock occurrences most of which are Phanerozoic in age. This secular distribution may be only apparent, however, because the number of Precambrian carbonatites is continually increasing along with the progress of research in shield areas. We describe here two Precambrian carbonatites from Finland which were discovered and drilled by Rautaruukki Co already in 1971-1972 but were reported so briefly in the international literature that their carbonatite origin has been doubted (Kresten et al. 1977, p. 64, cf. Woolley 1989). This paper provides new petrographic, mineralogical and geochemical data which prove that the early prospectors of the Rautaruukki Co were right when they classified the Kortejärvi and Laivajoki occurrences among real carbonatites (Nykänen 1993).

The Kortejärvi and Laivajärvi carbonatites are unexposed, metamorphosed and deformed, all features which hamper their investigation. The available drill core material suggests that they are unusual in that they are not associated with alkaline rocks and that no fenites are encountered.

\section{GEOLOGICAL SETTING}

The Kortejärvi carbonatite and Laivajärvi carbonatite are met with within the c. $1850 \mathrm{Ma}$ old (Svecokarelian) Hirvaskoski Shear Zone which separates the Archaean Pudasjärvi Complex from the Archaean Kuhmo Complex in northern Finland (Kärki et al. 1993) (Fig. 1). The country bedrock consists of psammitic gneisses and amphibolites (Honkamo 1979), which represent intensely deformed early Proterozoic (c. $2400 \mathrm{Ma}$ ) metalavas and associated turbiditic metapsammites of the Sumi-Sariola tectofacies (Laajoki 1991) or Silvennoinen's (1991) greenstone I. 


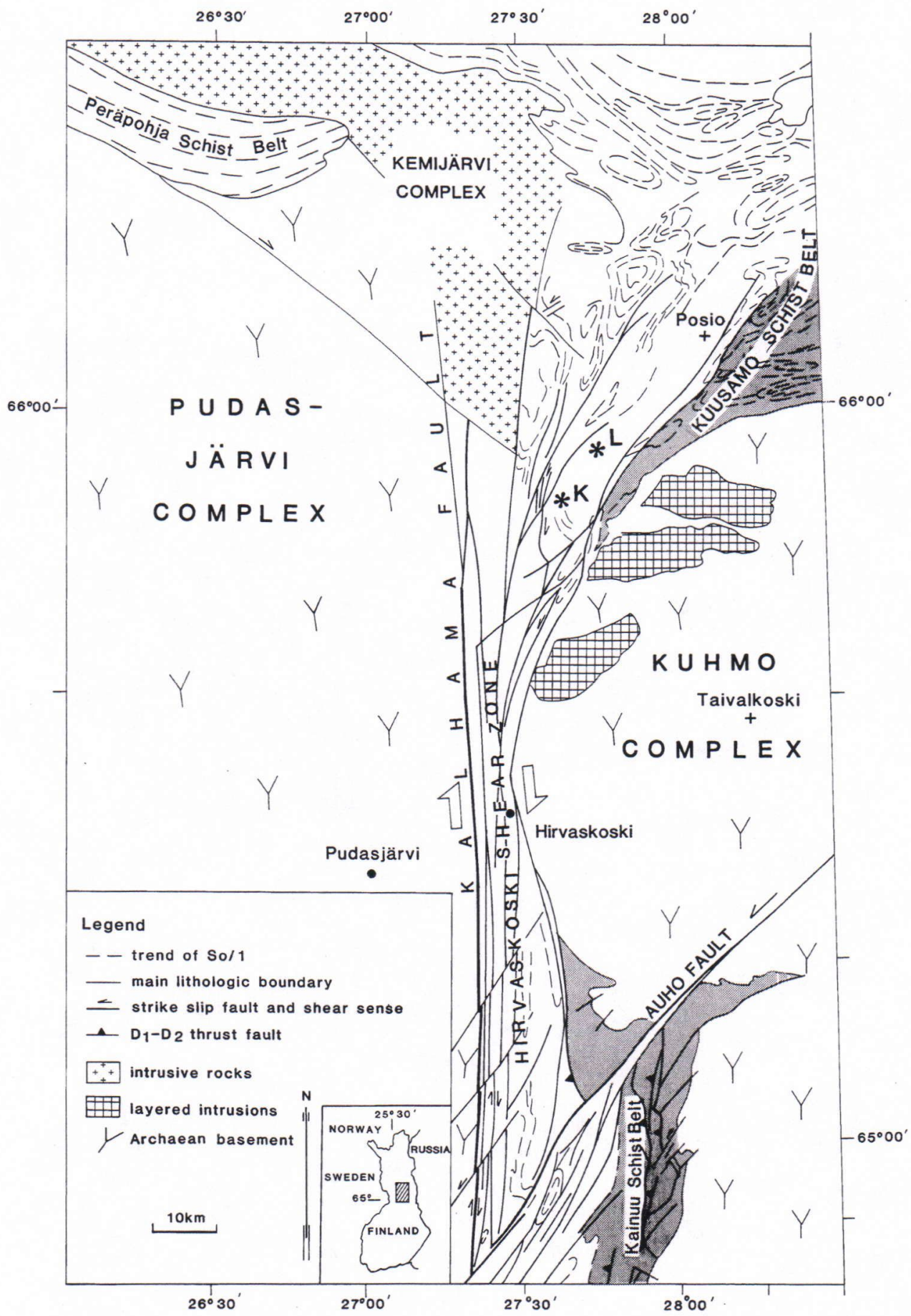

Fig. 1. Location of the Kortejärvi $(K)$ and Laivajoki $(L)$ occurrences within the Hirvaskoski shear zone, Taivalkoski and Posio areas. Map from Kärki et al. 1993. 
Evidence from two deep drill holes and various geophysical maps (Fig. 2) indicates that the Kortejärvi carbonatite forms a body about 30-60 $\mathrm{m}$ wide and $2000 \mathrm{~m}$ long which dips steeply to the east, while the Laivajärvi carbonatite (observed in four drill holes) forms with its southern extension (the Petäikkö-Suvantovaara anomaly), which is undrilled, but verified by till geochemistry, an occurrence $20 \mathrm{~m}$ wide and $4000 \mathrm{~m}$ long which dips $60^{\circ}$ to the southeast. The present geometries of these occurrences are secondary, being attributable to the high strain associated with the creation of the Hirvaskoski Shear Zone (Kärki et al. 1993).

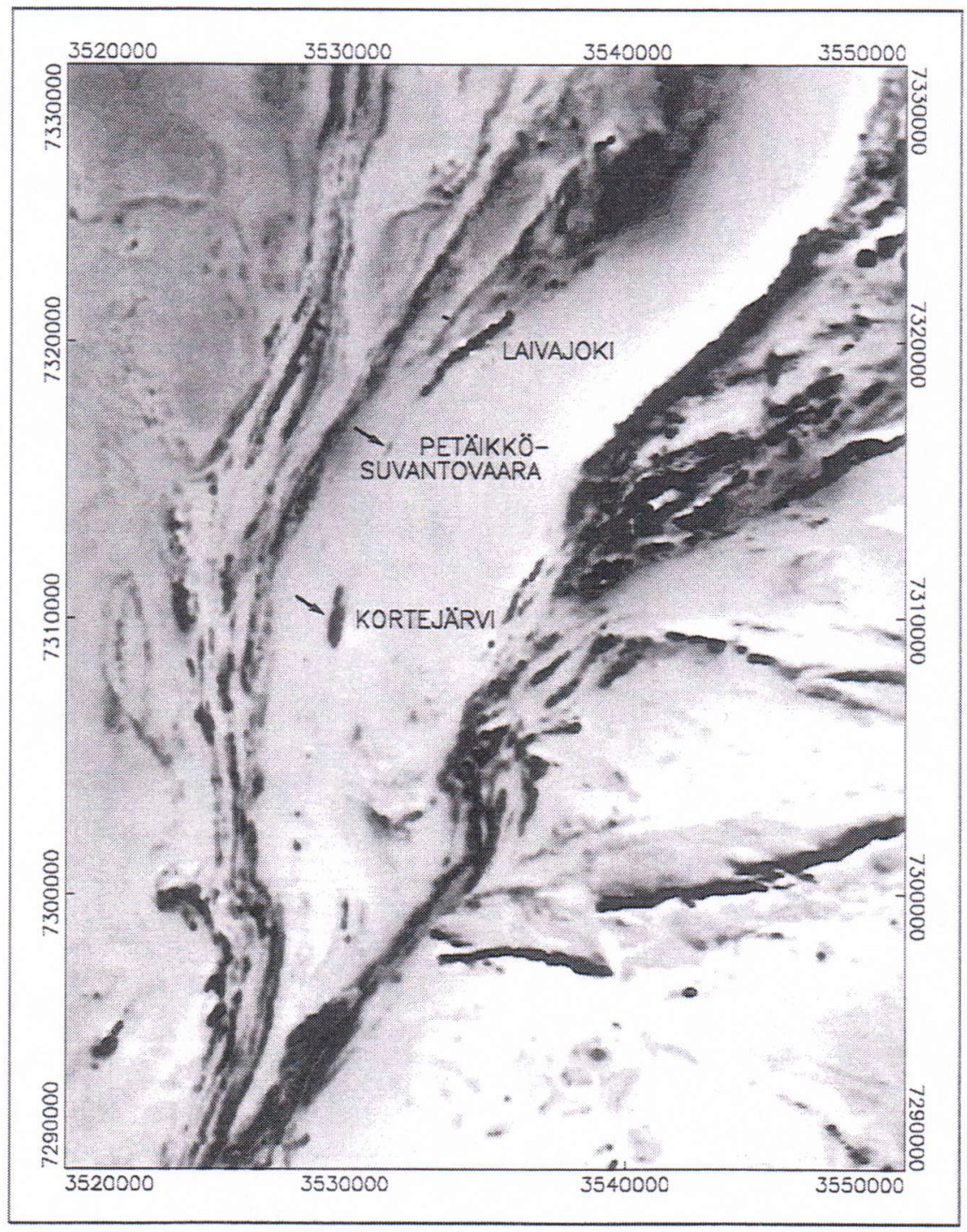

Fig. 2. Location of the Kortejärvi and Laivajoki occurrences and the propable carbonatite occurence of Petäikkö-Suvantovaara on the aeromagnetic total field map of the Hirvaskoski shear zone. Low-altitude (c. $35 \mathrm{~m}$ ) data from the Geological Survey of Finland. Dark anomalies are positive. Map area $30 \times 40 \mathrm{~km}$. 
The phlogopite of the Kortejärvi carbonatite gives a K-Ar age of $1875 \pm 75 \mathrm{Ma}$ (Kresten et al. 1977), which is considered indicative of the age of the Svecokarelian amphibolite-facies metamorphism, whereas a preliminary U-Pb zircon age of $2020 \mathrm{Ma}$ has been reported for the Laivajärvi carbonatite, (personal communication by O. Kouvo in Vartiainen \& Woolley 1974). Analytical data for this determination have not been published. In further studies the isotope systematics of zircon in the Kortejärvi and Laivajoki carbonatites has turned out to be complex, and it appears that the age of 2020 Ma represents a maximum estimate for the crystallization of these intrusions (Karhu and Mänttäri, in prep.).

\section{PETROGRAPHY OF THE KORTEJÄRVI OCCURRENCE}

We employ here the carbonatite nomenclature of Woolley and Kempe (1989) with prefix $a b$ brevitions $c$ - and $d$-for calcite and dolomite, respectively.

The main rock types of the Kortejärvi carbonatite are c-carbonatite, which makes up about $50 \%$ of the drill sections, and d-carbonatite, which occupies the southern part of the intrusion. Glimmerite or phologopite rock and olivine - magnetite rock are met with only in thin bands (Fig. 3).

The c-carbonatite is a banded, mediumgraded, bluish or white rock (Fig. 4) and mostly contains over $95 \%$ carbonates. Accessory minerals include dolomite, magnetite, inverse-pleochroic phlogopite, olivine, serpentine, tremolite, actinolite, apatite and the REE minerals allanite and monazite. Both carbonates are exsolutionfree. Locally the rock can include over $10 \%$ dolomite and is classified among the d-c-carbonatites. A zone of siderite-bearing d-c-carbonatite $2 \mathrm{~m}$ wide was met with in one drill hole.
The $d$-carbonatite is a yellowish rock with calcite, magnetite, normal or inverse-pleochroic phlogopite, allanite, zircon and apatite as accessory minerals. The amount of apatite is markedly greater (Fig. 5) than in the c-carbonatite, whereas calcite may rise to over $10 \%$, whereupon the rock is termed a c-d-carbonatite.

The olivine-magnetite rock is closely associated with the c-carbonatites but contains less than $50 \%$ carbonates. The main minerals are magnetite, olivine and calcite. Olivine is often altered to serpentine, iddingsite and bowlingite. Accessory minerals include dolomite, inversepleochroic phlogopite, K-richterite and zircon.

Glimmerite occurs in bands $0.1-4 \mathrm{~m}$ thick. It is mostly an almost monomineralic, dark green or brownish green phlogopite rock, but may also contain fresh actinolite and edenite. The phlogopite is normally pleochroic and the flakes are 0.4-1.8 mm long. Calcite, dolomite and apatite are the most common accessory minerals, with minor diopside, zircon, allanite, sulphides and magnetite.

\section{PETROGRAPHY OF THE LAIVAJOKI OCCURRENCE}

The Laivajärvi carbonatite differs from the Kortejärvi occurrence in that its c-carbonatites and d-carbonatites are rich in tremolite and that, in addition to these main rock types (Fig. 6), it also contains serpentine-talc-dolomite rock, while only a modest amount of glimmerite is present.

The Laivajärvi c-carbonatite is almost pure calcite carbonate rock with occasional dolomite. The accessory minerals include apatite, magnetite, phlogopite, tremolite (follows carbonates), actinolite (follows phlogopite), allanite, zircon and sulphides, but olivine and serpentine are lacking. 
10 Juha Nykänen et al.

\section{W \\ W}
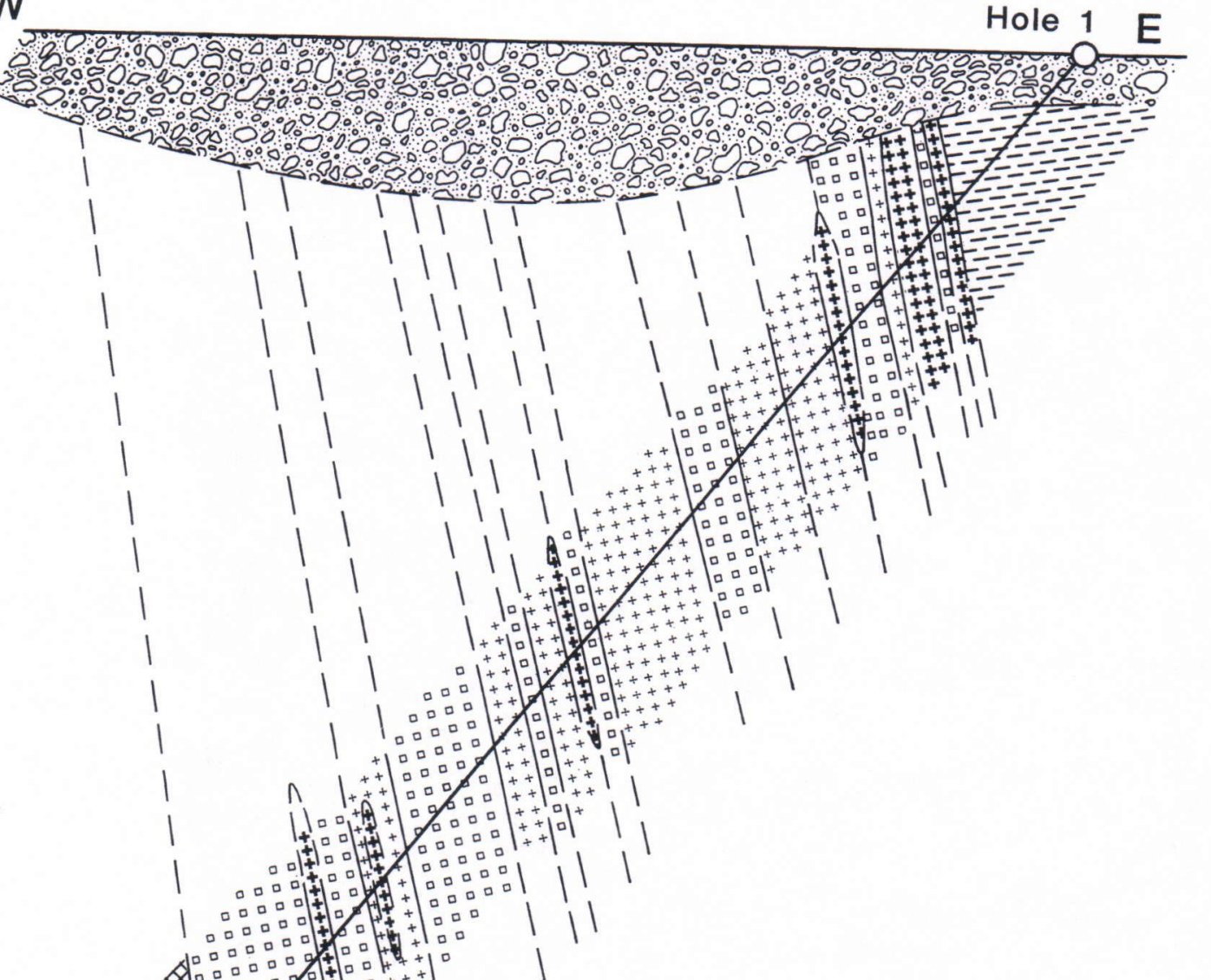

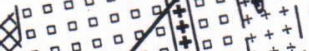

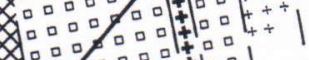

o 00 a 0 a

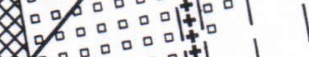

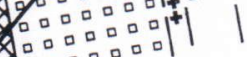

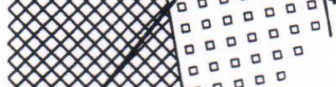

$x_{0}^{\circ} 0000$

$\begin{array}{lll}0 & 0 & 0\end{array}$

100

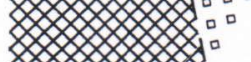

$++++$

Calcite carbonatite and dolomite-calcite carbonatite

Dolomite carbonatite and calcite-dolomite carbonatite

++++
+++

Amphibolite

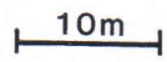

E- Quartz-feldspar schist

0:0: Overburden

Drill hole

Fig. 3. Lithology of the Kortejärvi occurrence as revealed by drill hole No. 1. Notice that repetition of the rock units may, at least in part, be due to deformation. Near-by outcrop observations indicate that the enclosing amphibolites are early Proterozoic metalavas. 


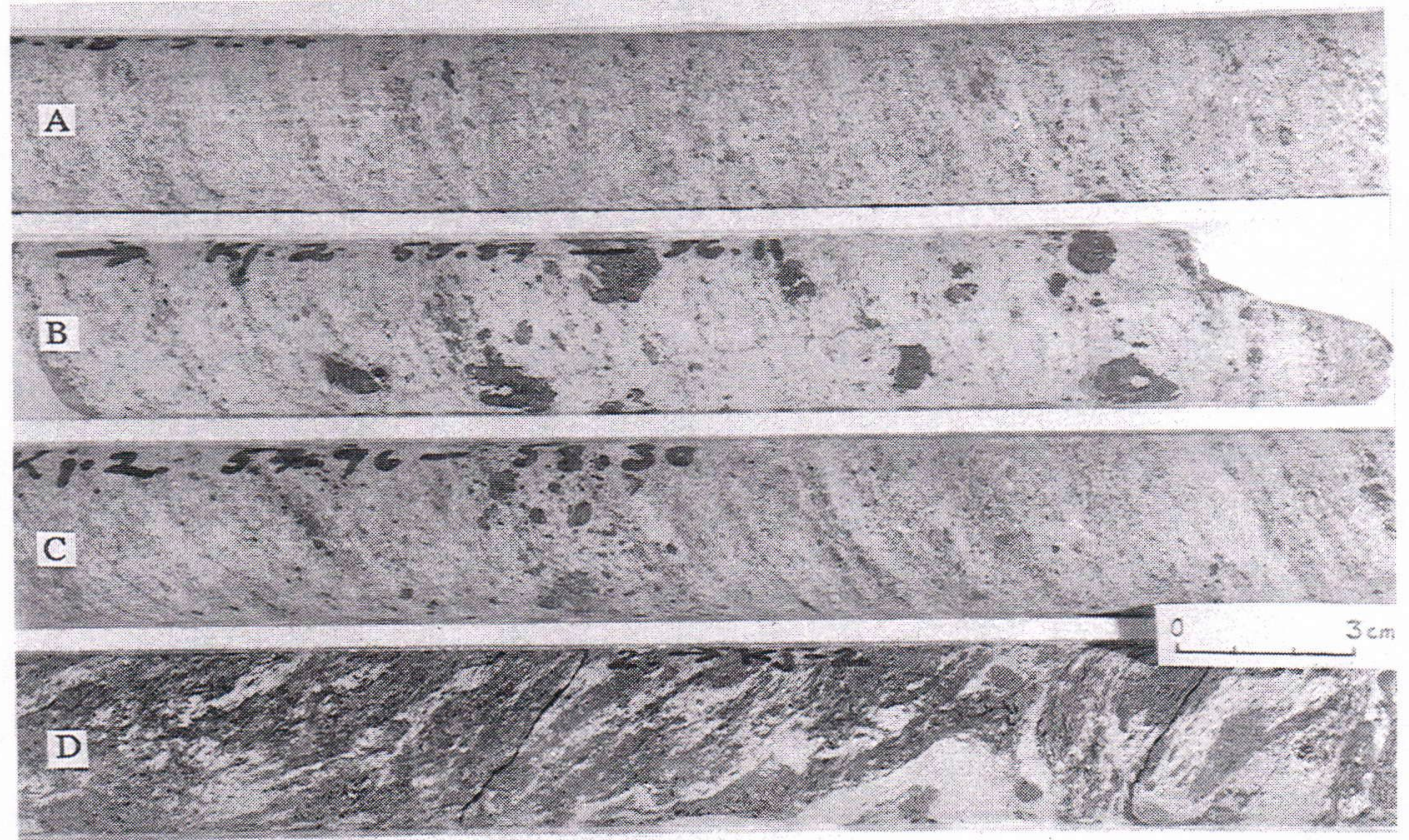

Fig. 4. Samples from drill core 2 of the Kortejärvi occurrence. A-C) Calcite-carbonatite. Dark grains are olivine. D) Glimmerite with calcite stripes. Banding in all samples is tectonic, not magmatic. Photos J. Nykänen.
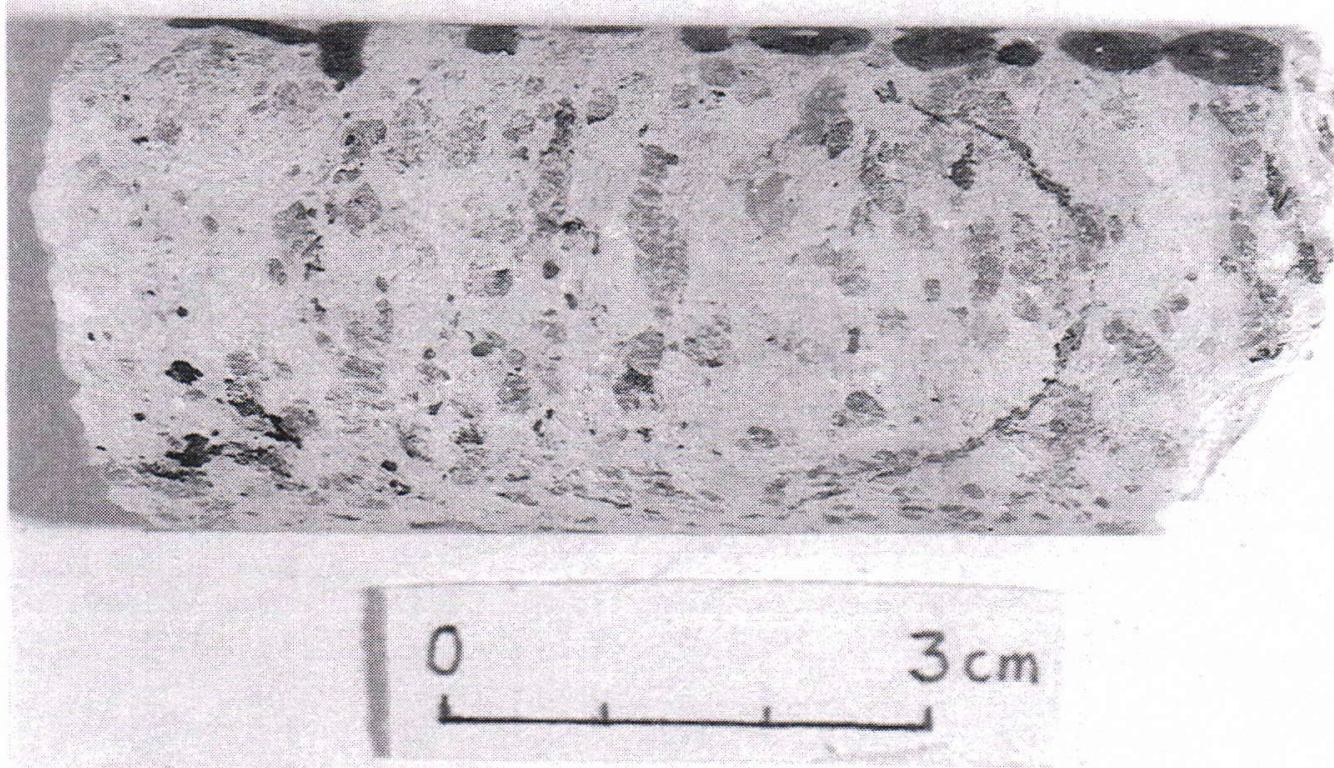

Fig. 5. Dolomite-carbonatite rich in apatite (light grey). Kortejärvi, drill core 2/108.00 m. Photo J. Nykänen. 


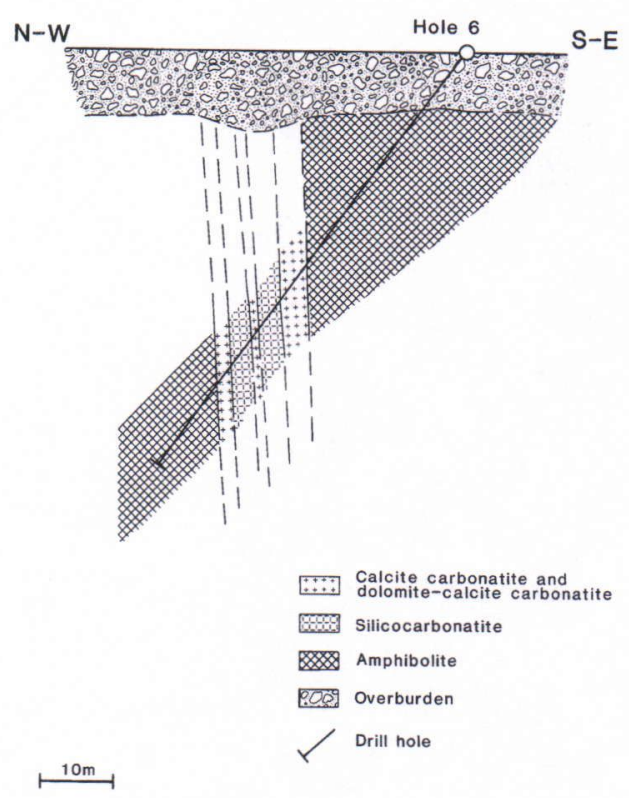

Fig. 6. Lithology of the Laivajoki occurrence as revealed by drill hole No. 6. Near-by outcrop observations indicate that amphibolites are early Proterozoic metalavas and quartz-feldspar schists with associated turbiditic metasedimentary rocks.

The carbonatites rich in tremolite are mostly tremolite-c-carbonatites, but dolomite-rich varieties do also occur. The silicate:carbonate ratio varies greatly, but the amount of carbonates is over $50 \%$ on average, and thus the rocks are classified as the carbonatites. The largest tremolite crystals may contain relics of diopside in their core zones, the only manner in which pyroxene occurs at the Laivajärvi site. Magnetite is fairly abundant and occurs in spots or thin bands. Accessory minerals are phlogopite, allanite and zircon. Zircon is abundant by comparision with the other rocks, and follows tremolite (cf. Kapustin 1980).

In addition to the minerals which give it its name, the serpentine-talc-dolomite rock contains tremolite, phologopite and magnetite as its main minerals. The basic difference relative to the tremolite-c-carbonatites is the occurrence of ser- pentine in spots c. $2 \mathrm{~mm}$ in diameter and smallscale talc, which both represent alteration products of olivine.

Glimmerite is rare at Laivajoki and consists almost solely of normally pleochroic phlogopite with magnetite, pyrite and apatite as common accessory minerals.

\section{MINERALOGY AND MINERAL CHEM- ISTRY OF THE KORTEJÄRVI AND LAIVAJOKI OCCURRENCES}

Calcite is the most common mineral in both occurrences, and contains a little magnesium (MgO 0.23-2.3\%) and iron (0.08-1.56 \%) (Table 1 and Fig. 7). Dolomite is the most common $\mathrm{Fe}-\mathrm{Mg}$ carbonate, but is rimmed by ankerite in Laivajoki. The amount of iron in the zoned dolomites increases towards the rim (cf. Gittins 1989). Staining proved that the dolomite does not contain calcite exsolutions and vice versa. Siderite, which is relatively rare in carbonatites and belongs normally to magnesite-siderite series (Woolley and Buckley 1993), is occasionally met with at Kortejärvi and is calcic (5.25\% $\mathrm{CaO}$ ). It is not clear if the siderite is magmatic or a product of subsolidus - metamorphic processes. The chemical compositions of $\mathrm{Mg}-\mathrm{Fe}$ carbonates are given in Table 2 and Fig. 7. The dolomite of the olivine-magnetite rock contains the least $\mathrm{FeO}$, which indicates that it intruded during Sokolov's (1985) stage II before the cand d-carbonatites. EDS studies show that the Kortejärvi and Laivajärvi carbonates do not contain REEs.

Phlogopite is one of the main minerals in glimmerites and a common accessory mineral in other rocks. Its pleochroism is normal and weak in almost all the Laivajärvi rocks and the Kortejärvi glimmerite, but some of the Kortejärvi carbonatites and the olivine- magnetite rock may contain reverse-pleochroic tetraferriphlogopite. Both phlogopites are fresh and contain tiny zircon inclusions. Some of the Kortejärvi tertaferriphlogopites are rimmed by phlogopite. 
Table 1. Chemical compositions of the calcites from the Kortejärvi (Nos. 1-10) and Laivajoki (Nos. 11-15) carbonatites, Finland. Analyst. J. Nykänen.

\begin{tabular}{|c|c|c|c|c|c|c|c|c|c|c|}
\hline & 1 & 2 & 3 & 4 & 5 & 6 & 7 & 8 & 9 & 10 \\
\hline $\mathrm{MgO}$ & 1.63 & 1.60 & 0.28 & 0.64 & 1.35 & 0.65 & 1.45 & 0.38 & 0.44 & 0.23 \\
\hline $\mathrm{FeO}$ & 0.45 & 0.55 & 0.34 & 0.30 & 0.28 & 0.11 & 0.39 & 0.45 & 0.21 & 0.08 \\
\hline $\mathrm{MnO}$ & 0.19 & 0.21 & 0.20 & 0.15 & 0.11 & 0.09 & 0.15 & 0.21 & 0.09 & 0.53 \\
\hline $\mathrm{CaO}$ & 54.75 & 56.09 & 53.28 & 55.32 & 56.57 & 53.08 & 50.25 & 54.42 & 52.37 & 54.42 \\
\hline $\mathrm{CO}_{2}$ & 42.98 & 41.55 & 45.90 & 43.58 & 41.69 & 46.07 & 47.77 & 44.55 & 46.89 & 44.75 \\
\hline Total & 100.00 & 100.00 & 100.00 & 99.99 & 100.00 & 100.00 & 100.01 & 100.01 & 100.00 & 100.01 \\
\hline
\end{tabular}

Number of cations per 18 oxygen atoms

$\begin{array}{lllllllllll}\mathrm{Mg} & 0.2365 & 0.2267 & 0.0432 & 0.0944 & 0.1918 & 0.1002 & 0.2297 & 0.0572 & 0.0690 & 0.0348 \\ \mathrm{Fe} & 0.0366 & 0.0437 & 0.0294 & 0.0248 & 0.0223 & 0.0095 & 0.0347 & 0.0380 & 0.0185 & 0.0068 \\ \mathrm{Mn} & 0.0157 & 0.0169 & 0.0175 & 0.0126 & 0.0089 & 0.0079 & 0.0135 & 0.0180 & 0.0080 & 0.0455 \\ \mathrm{Ca} & 5.7112 & 5.7127 & 5.9098 & 5.8681 & \text { s.mo } & 5.8824 & 5.7221 & 5.8869 & 5.9045 & 5.9129 \\ \mathrm{C} & \text { n.d. } & \text { n.d. } & \text { n.d. } & \text { n.d. } & \text { n.d. } & \text { n.d. } & \text { n.d. } & \text { n.d. } & \text { n.d. } & \text { n.d. }\end{array}$

n.d. $=$ not determined

\begin{tabular}{lrrrrr} 
& \multicolumn{1}{c}{11} & \multicolumn{1}{c}{12} & \multicolumn{1}{c}{13} & \multicolumn{1}{c}{14} & \multicolumn{1}{c}{15} \\
\hline $\mathrm{MgO}$ & 1.22 & 1.18 & 1.24 & 2.30 & 1.36 \\
$\mathrm{FeO}$ & 0.73 & 1.06 & 0.87 & 1.56 & 0.74 \\
$\mathrm{MnO}$ & 0.36 & 0.34 & 0.37 & 0.31 & 0.53 \\
$\mathrm{CaO}$ & 52.29 & 54.78 & 51.35 & 50.45 & 59.94 \\
$\mathrm{CO}_{2}$ & 45.40 & 42.65 & 46.17 & 45.38 & 37.43 \\
\hline $\mathrm{Total}$ & 100.00 & 100.01 & 100.00 & 100.00 & 100.00 \\
& & & & & \\
$\mathrm{Mg}$ & 0.1857 & 0.1712 & 0.1915 & 0.3483 & 0.1807 \\
$\mathrm{Fe}$ & 0.0623 & 0.0863 & 0.0754 & 0.1326 & 0.0552 \\
$\mathrm{Mn}$ & 0.0311 & 0.0280 & 0.0325 & 0.0267 & 0.0400 \\
$\mathrm{Ca}$ & 5.7208 & 5.7144 & 5.7006 & 5.4924 & 5.7241 \\
$\mathrm{C}$ & n.d. & n.d. & n.d. & nd & nd.
\end{tabular}

Rock type, drill hole number/depth (m)

$1=$ Calcite carbonatite $1 / 56.20$

$2=$ Calcite carbonatite $1 / 56.20$

3 = Calcite-dolomite carbonatite 1/93.40

$4=$ Dolomite carbonatite $1 / 79.40$ (light)

$5=$ Dolomite carbonatite $1 / 79.40$ (red)

$6=$ Olivine-magnetite rock $1 / 37.00$ (light)

$7=$ Olivine-magnetite rock $1 / 37.00$ (red)

$8=$ Olivine-magnetite rock $1 / 37.00$ (light)

$9=$ Olivine-magnetite rock $1 / 37.00$ (red)
$10=$ Olivine-magnetite rock $2 / 52.70$

$11=$ Calcite carbonatite $5 / 60.25$

$12=$ Dolomite-calcite carbonatite $6 / 47.40$

$13=$ Dolomite-calcite carbonatite $6 / 47.40$

$14=$ Dolomite-calcite carbonatite $6 / 4740$

$15=$ Tremolite-dolomite carbonatite $3 / 75.60$ 
14 Juha Nykänen et al.

Table 2. Chemical compositions of Mg-Fe carbonates of the Kortejärvi (Nos. 1-11\& 21) and Laivajoki (Nos. 12-20) carbonatites, Finland. Analyst. J. Nykänen.

\begin{tabular}{lrrrrrrrrrrr} 
& \multicolumn{1}{c}{1} & \multicolumn{1}{c}{2} & \multicolumn{1}{c}{3} & \multicolumn{1}{c}{4} & \multicolumn{1}{c}{5} & \multicolumn{1}{c}{6} & \multicolumn{1}{c}{7} & \multicolumn{1}{c}{8} & \multicolumn{1}{c}{9} & \multicolumn{1}{c}{10} & \multicolumn{1}{c}{11} \\
\hline $\mathrm{FeO}$ & 3.40 & 3.26 & 4.24 & 4.47 & 4.29 & 2.68 & 1.88 & 2.05 & 2.95 & 2.34 & 2.32 \\
$\mathrm{MnO}$ & 0.16 & 0.18 & 0.25 & 0.25 & 0.33 & 0.18 & 0.15 & 0.14 & 0.19 & 0.47 & 0.25 \\
$\mathrm{MgO}$ & 18.36 & 18.93 & 17.99 & 18.93 & 17.96 & 18.55 & 19.64 & 19.77 & 18.77 & 15.82 & 19.11 \\
$\mathrm{CaO}$ & 28.84 & 29.14 & 28.43 & 29.83 & 28.10 & 2838 & 29.28 & 28.61 & 29.11 & 33.41 & 29.74 \\
$\mathrm{CO}_{2}$ & 49.24 & 48.49 & 49.09 & 46.52 & 49.32 & 50.22 & 49.05 & 49.43 & 48.99 & 47.96 & 48.58 \\
Total & 100.00 & 100.00 & 100.00 & 100.00 & 100.00 & 100.01 & 100.00 & 100.00 & 100.01 & 100.00 & 100.00
\end{tabular}

Number of cations per 18 oxygen atoms

$\begin{array}{llllllllllll}\mathrm{Mg} & 2.6810 & 2.7167 & 2.6361 & 2.6400 & 2.6442 & 2.7443 & 2.8173 & 2.8538 & 2.7165 & 2.2919 & 2.7345 \\ \mathrm{Fe} & 0.2786 & 0.2625 & 0.3486 & 0.3498 & 0.3544 & 0.2225 & 0.1513 & 0.1660 & 0.2395 & 01902 & 0.1863 \\ \mathrm{Mn} & 0.0133 & 0.0147 & 0.0208 & 0.0198 & 00276 & 0.0151 & 0.0122 & 0.0115 & 0.0156 & 00387 & 00203 \\ \mathrm{Ca} & 3.0272 & 3.0061 & 2.9945 & 2.9904 & 2.9738 & 3.0181 & 3.0192 & 2.9687 & 3.0284 & 3.4792 & 3.0590 \\ \mathrm{C} & \text { n.d. } & \text { n.d. } & \text { n.d. } & \text { n.d. } & \text { n.d. } & \text { n.d. } & \text { n.d. } & \text { n.d. } & \text { n.d. } & \text { n.d. } & \text { n.d. }\end{array}$

n.d. $=$ not determined

\begin{tabular}{lrrrrrrrrrr} 
& 12 & \multicolumn{1}{c}{13} & \multicolumn{1}{c}{14} & \multicolumn{1}{c}{15} & \multicolumn{1}{c}{16} & 17 & 18 & \multicolumn{1}{c}{19} & \multicolumn{1}{c}{20} & \multicolumn{1}{c}{21} \\
\hline $\mathrm{FeO}$ & 3.99 & 3.74 & 3.42 & 3.72 & 5.58 & 8.83 & 7.75 & 4.71 & 3.64 & 53.22 \\
$\mathrm{MnO}$ & 0.38 & 0.32 & 0.31 & 0.32 & 0.41 & 0.43 & 0.44 & 0.56 & 0.23 & 0.29 \\
$\mathrm{MgO}$ & 18.40 & 17.72 & 18.25 & 17.95 & 16.51 & 14.79 & 15.57 & 20.30 & 18.86 & 0.71 \\
$\mathrm{CaO}$ & 29.11 & 28.24 & 28.83 & 28.14 & 28.33 & 28.28 & 28.20 & 31.08 & 28.60 & 5.25 \\
$\mathrm{CO}$ & 48.12 & 49.99 & 49.20 & 49.87 & 49.17 & 47.66 & 48.04 & 43.36 & 48.67 & 40.54 \\
\hline Total & 100.00 & 100.01 & 100.01 & 100.00 & 100.00 & 99.99 & 100.00 & 100.01 & 100.00 & 100.01 \\
& & & & & & & & & & \\
$\mathrm{Mg}$ & 2.6424 & 2.6382 & 2.6663 & 2.6628 & 2.4618 & 2.2011 & 2.3101 & 2.6709 & 2.7208 & 0.1234 \\
$\mathrm{Fe}$ & 0.3215 & 0.3124 & 0.2803 & 0.3096 & 0.4668 & 0.7373 & 0.6452 & 0.3477 & 0.2946 & 5.1918 \\
$\mathrm{Mn}$ & 0.0310 & 0.0271 & 0.0257 & 0.0270 & 0.0347 & 0.0364 & 0.0371 & 0.0419 & 0.0189 & 0.0287 \\
$\mathrm{Ca}$ & 3.0051 & 3.0223 & 3.0277 & 3.0006 & 3.0366 & 3.0253 & 3.0076 & 2.9395 & 2.9658 & 0.6561 \\
$\mathrm{C}$ & n.d. & n.d. & n.d. & n.d. & n.d. & n.d. & n.d. & nd & n.d. & n.d.
\end{tabular}

Carbonate, rock type, drill hole number/depth $(\mathrm{m})(\mathrm{Kj}=$ Kortejärvi, $\mathrm{Lj}=$ Laivajoki)

1-2 = dolomite, calcite carbonatite,

3 = dolomite, calcite-dolomite-carbonatite

4-5 = dolomite, dolomite carbonatite,

$6=$ dolomite, dolomite carbonatite,

$7-8=$ dolomite, olivine-magnetite rock,

$9=$ dolomite, olivine-magnetite rock,

10-11 = dolomite, olivine-magnetite rock,

12 = dolomite, calcite-carbonatite,

13-14 = dolomite, dolomite-calcite-carbonatite,

15-16 = dolomite, dolomite-calcite-carbonatite,

17 = ankerite, dolomite-calcite-carbonatite,
Kj 156.20

Kj 193.40

Kj 118.40

Kj 179.40

Kj 137.00

Kj 143.00

$\mathrm{Kj} 252.70$

Lj 560.25

Lj 647.40

Lj 647.40

Lj 647.40

(Nos. 15-17 are from the same grain, No. 15 from its center, No. 17 from the rim)

$18=$ ankerite, dolomite-calcite-carbonatite, $\quad \mathrm{Lj} 647.40$

$19=$ dolomite, tremolite-dolomite carbonatite, $\quad \operatorname{Lj} 375.60$

$20=$ dolomite, serpentine-talc-dolomite rock, $\quad \operatorname{Lj} 644.40$

21 = siderite, calcite-dolomite carbonatite $\quad$ Kj 193.40 


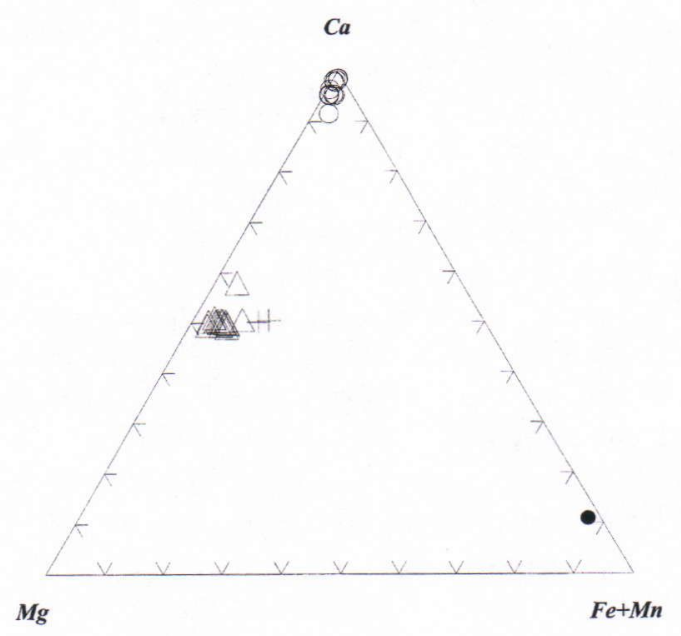

Fig. 7. Compositions of the Kortejärvi and Laivajoki carbonates $($ Tables 1 and 2). Open circle $=$ calcites $(N=15)$. Triangles $=$ dolomites $(N=16)$. Cross $=$ ankerite $(N=2)$. Filled circle $=$ siderite $(N=1)$.

Only the Kortejärvi phlogopites were analysed. Like the Jacupiranga micas (Gaspar and Wyllie 1987, their Fig. 5), they all appeared to be low in titanium $\left(0.06-0.49 \% \mathrm{TiO}_{2}\right)$, rich in $\mathrm{MgO}$ and their $\mathrm{TiO}_{2}$ contents increase with decreasing $\mathrm{MgO}$ (Table 3). The micas of the olivine-magnetite rock are less evolved than those of the d-carbonatites, whereas the phlogopite of the Kortejärvi glimmerite is lowest in $\mathrm{MgO}$.

Fresh olivine is met with only at Kortejärvi, where it occurs in thin olivine-magnetite bands and occasionally also in c-carbonatite and d-carbonatite. Most of it has been altered to serpentine, iddingsite, bowlingite, magnetite or even clinohumite. The olivine is a little richer in magnesium in the olivine-magnetite rocks than it is in the carbonatites (Table 4).

The clinohumite is fairly rich in titanium (2.45-2.89 wt.\% $\mathrm{TiO}_{2}$, Table 4) and, like the Jacupiranga clinohumite, which is consider by Gaspar (1992) metasomatic, is enriched in $\mathrm{MgO}$ by comparison with the olivine. It is rich in $\mathrm{FeO}$, however, and in this respect resembles the clinohumite of Cargill Lake (Kocman and Ruck- lidge 1973). The chemical compositions of the other alteration products of olivine (serpentine, bowlingite and iddingsite) can be found in Nykänen's (1993) theses.

Table 3. Chemical compositions of the phlogopites of the Kortejärvi carbonatite, Finland. Analyst. J. Nykänen.

\begin{tabular}{|c|c|c|c|c|c|c|c|}
\hline & 1 & 2 & 3 & 4 & 5 & 6 & 7 \\
\hline $\mathrm{SiO}_{2}$ & 43.20 & 42.03 & 41.77 & 41.87 & 41.96 & 40.79 & 40.49 \\
\hline $\mathrm{TiO}_{2}$ & 0.49 & 0.34 & 0.06 & 0.10 & 0.13 & 0.10 & 0.43 \\
\hline $\mathrm{Al}_{2} \mathrm{O}_{3}$ & 9.91 & 10.29 & 8.94 & 10.28 & 11.56 & 11.21 & 12.23 \\
\hline $\mathrm{Cr}_{2} \mathrm{O}_{3}$ & 0.00 & 0.02 & 0.00 & 0.00 & 0.00 & 0.00 & 0.06 \\
\hline $\mathrm{V}_{2} \mathrm{O}_{3}$ & 0.02 & 0.04 & 0.00 & 0.01 & 0.01 & 0.00 & 0.06 \\
\hline $\mathrm{FeO}$ & 9.94 & 11.19 & 10.70 & 4.87 & 3.66 & 4.48 & 11.31 \\
\hline $\mathrm{MnO}$ & 0.01 & 0.03 & 0.03 & 0.01 & 0.03 & 0.03 & 0.13 \\
\hline $\mathrm{MgO}$ & 22.61 & 22.23 & 23.47 & 27.03 & 25.50 & 25.97 & 19.83 \\
\hline $\mathrm{NiO}$ & 0.01 & 0.00 & 0.00 & 0.00 & 0.02 & 0.01 & 0.03 \\
\hline $\mathrm{ZnO}$ & 0.00 & 0.00 & 0.00 & 0.00 & 0.00 & 0.00 & 0.05 \\
\hline $\mathrm{CaO}$ & 0.00 & 0.00 & 0.01 & 0.01 & 0.03 & 0.03 & 0.08 \\
\hline $\mathrm{Na}_{2} \mathrm{O}$ & 0.01 & 0.01 & 0.21 & 0.37 & 0.53 & 0.44 & 0.72 \\
\hline $\mathrm{K}_{2} \mathrm{O}$ & 9.60 & 8.65 & 9.97 & 8.69 & 9.47 & 8.40 & 8.85 \\
\hline & 60 & 94.83 & 95.16 & 3.24 & 92.90 & 91.46 & 94.27 \\
\hline
\end{tabular}

Number of cations per 22 oxygen atoms

\begin{tabular}{|c|c|c|c|c|c|c|c|}
\hline $\mathrm{Si}$ & 6.2182 & 6.1285 & 6.1277 & 6.0459 & 6.0628 & 5.9884 & 5.9782 \\
\hline $\mathrm{Al}$ & 1.6811 & 1.7683 & 1.5457 & 1.7495 & 1.9686 & 1.9396 & 2.1281 \\
\hline $\mathrm{Ti}$ & 0.0530 & 0.0373 & 0.0066 & 0.0109 & 0.0141 & 0.0110 & 0.0477 \\
\hline $\mathrm{Cr}$ & 0.0000 & 0.0023 & 0.0000 & 0.0000 & 0.0000 & 0.0000 & 0.0070 \\
\hline V & 0.0023 & 0.0047 & 0.0000 & 0.0012 & 0.0012 & 0.0000 & 0.0071 \\
\hline $\mathrm{Fe}$ & 1.1965 & 1.3645 & 1.3127 & 0.5881 & 0.4423 & 0.5500 & 1.3965 \\
\hline Mn & 0.0012 & 0.0037 & 0.0037 & 0.0012 & 0.0037 & 0.0037 & 0.0163 \\
\hline $\mathrm{Mg}$ & 4.8508 & 4.8314 & 5.1320 & 5.8175 & 5.4918 & 5.6828 & 4.3639 \\
\hline $\mathrm{Ni}$ & 0.0012 & 0.0000 & 0.0000 & 0.0000 & 0.0023 & 0.0012 & 0.0036 \\
\hline $\mathrm{Zn}$ & 0.0000 & 0.0000 & 0.0000 & 0.0000 & 0.0000 & 0.0000 & 0.0055 \\
\hline $\mathrm{Ca}$ & 0.0000 & 0.0000 & 0.0016 & 0.0015 & 0.0046 & 0.0047 & 0.0127 \\
\hline $\mathrm{Na}$ & 0.0028 & 0.0028 & 0.0597 & 0.1036 & 0.1485 & 0.1252 & 0.2061 \\
\hline K & 1.7627 & 1.6089 & 1.8658 & 1.6007 & 1.7455 & 1.5731 & 1.6668 \\
\hline
\end{tabular}

1 = dolomite carbonatite $\mathrm{Kj} 118.40$ (core)

2 = dolomite carbonatite $\mathrm{Kj} 118.40$ (core)

3 = dolomite carbonatite $\mathrm{Kj} 175.85$

4 = olivine-magnetite rock $\mathrm{Kj} 137.00$

$\mathbf{5}$ = olivine-magnetite rock $\mathrm{Kj} 259.70$ (core)

6 = olivine-magnetite rock $\mathrm{Kj} 259.70$ (core)

7 = glimmerite $\mathrm{Kj} 191.60$

The Kortejärvi and Laivajärvi occurrences differ from other carbonatites in that their amphiboles are mostly calcic amphiboles (Table 5, nomenclature after Leake et al. 1997) and sodic-calcic amphiboles are met with only in accessory amounts. Actinolite and tremolite occur as accessory minerals in the Kortejärvi c-carbonatite and d-carbonatite, whereas actinolite and edenite rimmed by actinolite are met with as main minerals in the Kortejärvi glimmerite. 
Tremolite is one of the main minerals in the tremolite-c-carbonatite and tremolite-d-carbonatite in the Laivajärvi intrusion and an accessory mineral in the other rocks except for the glimmerite, which does not contain amphiboles at all. The only sodic-calcic amphibole detected is richterite, which occurs as inclusions in the olivine of the Kortejärvi olivine-magnetite rock and is fairly rich in alkalis $\left(\mathrm{Na}_{2} \mathrm{O} 4.56 \%, \mathrm{~K}_{2} \mathrm{O}\right.$ $2.06 \%)$.

Table 4. Chemical compositions of olivine (Nos. 1-4) and clinohumite (Nos. 5 and 6) from the Kortejärvi carbonatite, Finland. Analyst. J. Nykänen.

\begin{tabular}{lrrrrrr}
\multicolumn{1}{l}{1} & \multicolumn{1}{l}{2} & \multicolumn{1}{l}{3} & \multicolumn{1}{l}{4} & \multicolumn{1}{l}{5} \\
\hline $\mathrm{SiO}_{2}$ & 39.86 & 39.59 & 38.56 & 39.27 & 36.03 & 35.89 \\
$\mathrm{TiO}_{2}$ & 0.00 & 0.01 & 0.00 & 0.00 & 2.89 & 2.45 \\
$\mathrm{Al}_{2} \mathrm{O}_{3}$ & 0.00 & 0.00 & 0.00 & 0.02 & 0.00 & 0.01 \\
$\mathrm{Cr}_{2} \mathrm{O}_{3}$ & 0.02 & 0.00 & 0.00 & 002 & 0.00 & 0.02 \\
$\mathrm{~V}_{2} \mathrm{O}_{3}$ & 0.02 & 0.02 & 0.00 & 0.00 & 0.03 & 0.01 \\
$\mathrm{FeO}$ & 20.12 & 19.66 & 21.95 & 24.84 & 15.94 & 15.70 \\
$\mathrm{MnO}$ & 0.40 & 0.38 & 0.57 & 0.61 & 0.35 & 0.52 \\
$\mathrm{NiO}$ & 0.00 & 0.00 & 0.00 & 0.00 & 0.03 & 0.00 \\
$\mathrm{MgO}$ & 41.33 & 42.16 & 39.24 & 37.76 & 43.52 & 43.59 \\
$\mathrm{ZnO}$ & 0.00 & 0.02 & 0.07 & 0.05 & 0.00 & 0.05 \\
$\mathrm{CaO}$ & 0.01 & 0.02 & 0.01 & 0.00 & 0.02 & 0.05 \\
$\mathrm{Na} 2 \mathrm{O}$ & 0.01 & 0.00 & 0.01 & 0.02 & 0.00 & 0.00 \\
$\mathrm{~K} 2 \mathrm{O}$ & 0.00 & 000 & 0.00 & 0.00 & 0.00 & 0.00 \\
\hline $\mathrm{Total}$ & 101.77 & 101.86 & 100.41 & 102.59 & 98.81 & 98.29
\end{tabular}

Number of cations per 4 oxygen atoms

$\begin{array}{lllllll}\mathrm{Si} & 1.0056 & 0.9969 & 0.9982 & 1.0055 & 3.9521 & 3.9580 \\ \mathrm{Al} & 0.0000 & 0.0000 & 00000 & 0.0006 & 0.0000 & 0.0013 \\ \mathrm{Ti} & 0.0000 & 0.0002 & 0.0000 & 0.0000 & 0.2384 & 0.2032 \\ \mathrm{Cr} & 0.0004 & 0.0000 & 0.0000 & 0.0004 & 0.0000 & 0.0017 \\ \mathrm{~V} & 0.0004 & 0.0004 & 0.0000 & 0.0000 & 0.0026 & 0.0009 \\ \mathrm{Mg} & 1.5541 & 15823 & 1.5141 & 1.4410 & 7.1153 & 7.1652 \\ \mathrm{Ni} & 0.0000 & 0.0000 & 0.0000 & 0.0000 & 0.0026 & 0.0000 \\ \mathrm{Fe} & 0.4245 & 0.4140 & 0.4752 & 05319 & 1.4622 & 1.4480 \\ \mathrm{Mn} & 0.0085 & 0.0081 & 0.0125 & 0.0132 & 0.0325 & 0.0486 \\ \mathrm{Zn} & 0.0000 & 0.0004 & 0.0013 & 0.0009 & 0.0000 & 0.0041 \\ \mathrm{Ca} & 0.0003 & 0.0005 & 0.0003 & 0.0000 & 0.0024 & 0.0059 \\ \mathrm{Na} & 0.0005 & 0.0000 & 0.0005 & 0.0010 & 0.0000 & 0.0000 \\ \mathrm{~K} & 0.0000 & 0.0000 & 0.0000 & 0.0000 & 0.0000 & 0.0000\end{array}$

Rock, drill hole number/depth (m)

1 = olivine-magnetite rock $\mathrm{Kj} 143.00$

2 = olivine-magnetite rock $\mathrm{Kj} 148.60$

Fo 78.5

3 = calcite carbonatite $\mathrm{Kj} 271.40 \quad$ Fo 76.1

4 = calcite-dolomite carbonatite $\mathrm{Kj} 145.80$ Fo 73.0

5 = olivine-magnetite rock $\mathrm{Kj} 148.60$

6 = calcite carbonatite $\mathrm{Kj} 271.40$

Tremolite and actinolite are not listed into the minerals reported from carbonatites (Hogarth 1989, his Table 6.1). They have not been ana- lysed from any carbonatite occurence, only tentatively identified in many studies; e.g. Pell and Höy (1989) report them from the Blue River area, where the rocks have been metamorphosed in amphibolite facies.

Magnetite is most abundant in the Kortejärvi olivine-magnetite rock and in the Laivajärvi serpentine-talc-dolomite rock, but is also common elsewhere. It has almost stochiometric composition and is Ti-poor $\left(0.00-0.58 \% \mathrm{TiO}_{2}\right.$, Table 6), which are both features common to magnetites in carbonatites in general (Prins 1972, Mitchell 1979 ). The vanadium content is also rather low $\left(0.01-1.24 \% \mathrm{~V}_{2} \mathrm{O}_{3}\right)$.

Ilmenite occurs in the Kortejärvi and Laivajärvi magnetites as lamellae or inner or rim composite grains (see Buddington and Lindsley 1964, Haggerty 1991). The former are prominent at Kortejärvi, which points to oxidation exsolution origin, whereas ilmenite grains, some of which may be primary, are more common at Laivajoki. The $\mathrm{MgO}$ contents of the ilmenites in the carbonatites are in general low $(<1 \%$, Gasparin and Wyllie 1983, <5 \%, Mitchell 1986). In this light, the ilmenites of the Kortejärvi olivine-magnetite rocks and serpentinedolomite carbonatite are fairly rich in magnesium (4.14-7.13 and 3.04\% MgO, respectively, Table 7), whereas those in the c- and d-carbonatites have a typical low content.

Apatite is most abundant in the Kortejärvi d-carbonatite, but is also common in the c-carbonatites and glimmerites of both occurrences. It occurs in grains or grain aggregates $1-3 \mathrm{~mm}$ long. It is fluorapatite in both intrusions, containing 3.95-4.89 \% F and having a high $\mathrm{Sr} / \mathrm{Mn}$ ratio (33 on average) (cf. Hogarth 1989). No REE were detected in the EDS-studies.

Monazite (EDS identification) occurs in microcrystals rimming the apatite (Nykänen 1993, his Fig. 22), which points to REE-rich hydrothermal fluids (Mariano 1989a), or as inclusions in actinolite in the Laivajärvi c-d-carbonatite. Like the carbonatites in general (Mariano 1989b), the Kortejärvi and Laivajärvi monazites are poor in $\mathrm{CaO}$ (Nykänen 1993). 
Table 5. Chemical compositions and names (after Leake et. al 1997) of the amphiboles of the Kortejärvi (Nos. 1-6) and Laivajoki (Nos. 7-10) carbonatites, Finland. Analyst. J. Nykänen at the Department of Electron Optics, University of Oulu.

\begin{tabular}{|c|c|c|c|c|c|c|c|c|c|c|}
\hline Analyse No. & 1 & 2 & 3 & 4 & 5 & 6 & 7 & 8 & 9 & 10 \\
\hline Rock & Glimmerite & Ol-mgt-rock & C-carbonatite & Glimmerite & Glimmerite & C-carbonatite & Tre-d-carbonatite & Tre-d-carbonatite & Tre-d-carbonnatite & D-c-carbonatite \\
\hline Drill hole No. & $\mathrm{KJ} 1$ & K] 1 & KJ1 & $\mathrm{KJ} 1$ & KJ1 & KJ 2 & 니 3 & Lj3 & Lij5 & Lj6 \\
\hline Depth (m) & 15.35 & 43 & 56.2 & 91.6 & 91.6 & 71.4 & 75.6 & 75.61 & 50.85 & 47 \\
\hline Mineral name & Actinolite & Richterite & \begin{tabular}{|l|} 
Tremolite \\
\end{tabular} & Edenite (core) & Edenite (rim) & \begin{tabular}{l|l} 
Tremolite & \\
\end{tabular} & Tremolite & Tremolite & Tremolite & Actinolite \\
\hline SiO2 & 55.76 & 58.22 & 57.77 & 54.48 & 56.05 & 57.73 & 56.67| & 57.07 & 57.72 & 54.65 \\
\hline $\mathrm{Al}^{2} 203$ & 1.55 & 0.3 & 0.1 & 2.44 & 1.67 & 0.1 & 0.97 & 1.12 & 0.67 & 0.86 \\
\hline $\mathrm{FeO}$ & 7.19 & 2.63 & 2.5 & 8.38 & 8.35 & 2.78 & 4.92 & 4.3 & 3.36 & 6.61 \\
\hline $\mathrm{MgO}$ & 18.89 & 22.95 & 22.23 & 19.02 & 18.94 & 22.62 & 21.2 & 21.49 & 22.1 & 19.95 \\
\hline $\mathrm{CaO}$ & 12.01 & 8.32 & 10.02 & 8.59 & 10.53 & 12.2 & 12.71 & 12.6 & 11.63 & 9.64 \\
\hline $\mathrm{Na} 20$ & 0.47 & 4.56 & 2.86 & 3.87 & 2.73 & 0.92 & 0.38 & 0.42 & 1.17 & 2.04 \\
\hline K20 & 0.18 & 2.06 & 0.68 & 0.7 & 0.27 & 0.21 & 0.1 & 0.08 & 0.11 & 1.14 \\
\hline 102 & 0.02 & 0.14 & 0 & 0.14 & 0.02 & 0.07 & 0.04 & 0.06 & 0.06 & 0.12 \\
\hline Mno & 0.2 & 0.05 & 0.07 & 0.21 & 0.17 & 0.06 & 0.13 & 0.12 & 0.16 & 0.19 \\
\hline Total & 96.27 & 99.23 & 96.23 & 97.83 & 98.73 & 96.69 & 97.12 & 97.26 & 96.98 & 96.2 \\
\hline & & & & & & & & & & \\
\hline Ts! & 7.932 & 8.175 & 8.2 & 7.806 & 7.917 & 8.036 & 7.894 & 7.915 & 8.022 & 7.933 \\
\hline $\mathrm{TAl}$ & 0.068 & 0.022 & 0 & 0.194 & 0.099 & 0.008 & 0.106 & 0.085 & 0.028 & 0.072 \\
\hline TFe 3 & 0 & 0 & 0 & 0 & 0 & 0 & 0 & 0 & 0 & 0.032 \\
\hline TII & 0 & 0 & 0 & 0 & 0 & 0 & 0 & 0 & 0 & 0 \\
\hline sum $T$ & 8 & 8.197 & 8.2 & 8 & 8.016 & 8.044 & 8 & 8 & 8.049 & 8.038 \\
\hline & & & & & & & & & & \\
\hline CAI & 0.192 & \begin{tabular}{|c|}
0.027 \\
\end{tabular} & 0.017 & 0.217 & 0.179 & 0.008 & 0.053 & 0.098 & 0.082 & 0.075 \\
\hline $\mathrm{CF} Q 3$ & 0.085 & 0.004 & 0 & 0.295 & 0.096 & 0.051 & 0.102 & 0.097 & 0.114 & 0.233 \\
\hline $\mathrm{CtI}$ & 0.002 & 0.015 & 0 & 0.015 & 0.002 & 0.007 & 0.004 & 0.006 & 0.006 & 0.013 \\
\hline $\mathrm{CMg}$ & 4.006 & 4.804 & 4.704 & 4.063 & 3.988 & 4.694 & 4.402 & 4.443 & 4.579 & 4.317 \\
\hline$C F \theta 2$ & 0.703 & 0.147 & 0.245 & 0.397 & 0.725 & 0.236 & 0.431 & 0.349 & 0.21 & 0.35 \\
\hline $\mathrm{CMn}$ & 0.012 & 0.003 & 0.004 & 0.012 & 0.01 & 0.004 & 0.008 & 0.007 & 0.009 & 0.011 \\
\hline CCa & 0 & 0 & 0.03 & 0 & 0 & 0 & 0 & 0 & 0 & 0 \\
\hline Sum C & 5 & 5 & 5 & 5 & 5 & 5 & 5 & 5 & 5 & 5 \\
\hline & & & & & & & & & & \\
\hline BMg & 0 & 0 & 0 & 0 & 0 & 0 & 0 & 0 & 0 & 0 \\
\hline$B F=2$. & 0.067 & 0.158 & 0.051 & 0.312 & 0.165 & 0.037 & 0.04 & 0.053 & 0.067 & 0.187 \\
\hline BMn & 0.012 & 0.003 & 0.004 & 0.013 & 0.01 & 0.004 & 0.008 & 0.007 & 0.01 & 0.012 \\
\hline$B C A$ & 1.83 & 1.252 & 1.494 & 1.319 & 1.594 & 1.82 & 1.897 & 1.872 & 1.732 & 1.499 \\
\hline $\mathrm{BNa} \quad$ & 0.064 & 0.588 & 0.451 & 0.357 & 0.231 & 0.14 & 0.051 & 0.056 & 0.192 & 0.301 \\
\hline $\operatorname{Sum} B$ & 1.974 & 2 & 2 & 2 & 2 & 2 & 1.996 & 1.988 & 2 & 2 \\
\hline & & & & & & & & & & \\
\hline$A C a$ & 0 & 0 & 0 & 0 & 0 & 0 & 0 & 0 & 0 & 0 \\
\hline ANa & 0.065 & 0.654 & 0.336 & 0.718 & 0.517 & 0.108 & 0.052 & 0.057 & 0.124 & 0.273 \\
\hline$A K$ & 0.033 & 0.369 & 0.123 & 0.128 & 0.049 & 0.037 & 0.018 & 0.014 & 0.02 & 0.211 \\
\hline $\operatorname{sum} A$ & 0.098 & 1.023 & 0.46 & 0.846 & 0.566 & 0.146 & 0.069 & 0.071 & 0.143 & 0.484 \\
\hline Sum cat & 15.072 & 16.22 & 15.659 & 15.846 & 15582 & 15.19 & 15,065 & 15.059 & 15,192 & 15.522 \\
\hline sum oxy & 23.097 & 23.63 & 23.412 & 23.419 & 23.289 & 23.124 & 23.034 & 23.056 & \begin{tabular}{|l|l|}
23.165 \\
\end{tabular} & $\frac{10.022}{23.282}$ \\
\hline
\end{tabular}


Table 6. Chemical compositions of magnetites of the Kortejärvi (Nos. 1-5) and Laivajoki (Nos. 6-11) carbonatites, Finland. Analyst. J. Nykänen.

\begin{tabular}{|c|c|c|c|c|c|c|c|c|c|c|c|}
\hline & 1 & 2 & 3 & 4 & 5 & 6 & 7 & 8 & 9 & 10 & 11 \\
\hline $\mathrm{SiO}_{2}$ & 0.05 & 0.06 & 0.02 & 2.15 & 0.01 & 0.02 & 0.03 & 0.05 & 0.01 & 0.03 & 0.04 \\
\hline $\mathrm{TiO}_{2}$ & 0.09 & 0.29 & 0.49 & 0.00 & 0.39 & 0.07 & 0.37 & 0.26 & 0.08 & 0.19 & 0.58 \\
\hline $\mathrm{A}_{1} \mathrm{O}_{3}$ & 0.00 & 0.04 & 0.02 & 0.00 & 0.05 & 0.01 & 0.05 & 0.02 & 0.09 & 0.02 & 0.02 \\
\hline $\mathrm{Cr}_{2} \mathrm{O}_{3}$ & 0.05 & 0.02 & 0.01 & 0.00 & 0.04 & 0.28 & 0.00 & 0.00 & 0.01 & 0.02 & 0.01 \\
\hline $\mathrm{V}_{2} \mathrm{O}_{3}$ & 0.39 & 0.33 & 0.36 & 0.01 & 0.51 & 1.24 & 0.35 & 0.62 & 0.52 & 0.23 & 0.31 \\
\hline $\mathrm{Fe}_{2} \mathrm{O}_{3}$ & 66.99 & 67.22 & 65.50 & 65.47 & 68.61 & 69.47 & 69.42 & 69.16 & 66.39 & 67.20 & 66.89 \\
\hline $\mathrm{FeO}$ & 30.57 & 29.98 & 29.85 & 32.52 & 31.31 & 32.05 & 31.85 & 31.85 & 30.17 & 30.25 & 31.08 \\
\hline $\mathrm{MnO}$ & 0.00 & 0.06 & 0.06 & 0.08 & 0.07 & 0.04 & 0.04 & 0.00 & 0.01 & 0.07 & 0.04 \\
\hline $\mathrm{MgO}$ & 0.02 & 0.55 & 0.37 & 0.97 & 0.28 & 0.03 & 0.15 & 0.02 & 0.09 & 0.17 & 0.15 \\
\hline $\mathrm{ZnO}$ & 0.00 & 0.00 & 0.00 & 0.00 & 0.00 & 0.00 & 0.00 & 0.09 & 0.00 & 0.11 & 0.00 \\
\hline $\mathrm{NiO}$ & 0.00 & 0.00 & 0.00 & 0.00 & 0.00 & 0.00 & 0.19 & 0.02 & 0.00 & 0.00 & 0.00 \\
\hline $\mathrm{CaO}$ & 0.01 & 0.05 & 0.00 & 0.20 & 0.00 & 0.00 & 0.00 & 0.02 & 0.00 & 0.03 & 0.00 \\
\hline $\mathrm{K}_{2} \mathrm{O}$ & 0.00 & 000 & 000 & 0.03 & 0.00 & 0.00 & 0.00 & 0.00 & 0.00 & 0.00 & 0.01 \\
\hline Total & 98.19 & 98.63 & 96.69 & 101.44 & 101.27 & 103.21 & 102.47 & 102.11 & 97.36 & 98.37 & 99.13 \\
\hline
\end{tabular}

Cations per 6 oxygen atoms

$\begin{array}{llllllllllll}\mathrm{Si} & 0.0157 & 0.0187 & 0.0064 & 0.6420 & 0.0030 & 0.0060 & 0.0090 & 0.0151 & 0.0032 & 0.0094 & 0.0124 \\ \mathrm{Al} & 0.0000 & 0.0147 & 0.0075 & 0.0000 & 0.0179 & 0.0035 & 0.0177 & 0.0071 & 0.0335 & 0.0074 & 0.0073 \\ \mathrm{Cr} & 0.0124 & 0.0049 & 0.0025 & 0.0000 & 0.0096 & 0.0660 & 0.0000 & 0.0000 & 0.0025 & 0.0049 & 0.0025 \\ \mathrm{Fe} 3 & 15.815 & 15.723 & 15.654 & 14.711 & 15.667 & 15.593 & 15.689 & 15.695 & 15.790 & 15.818 & 15.619 \\ \mathrm{Ti} & 0.0212 & 0.0678 & 0.1170 & 0.0000 & 0.0890 & 0.0157 & 0.0836 & 0.0590 & 0.0190 & 0.0447 & 0.1353 \\ \mathrm{~V} & 0.0981 & 0.0822 & 0.0917 & 0.0024 & 0.1241 & 0.2966 & 0.0843 & 0.1499 & 0.1318 & 0.0577 & 0.0771 \\ \mathrm{Mg} & 0.0094 & 0.2548 & 0.1751 & 0.4317 & 0.1266 & 0.0133 & 0.0671 & 0.0090 & 0.0424 & 0.0793 & 0.0694 \\ \mathrm{Ni} & 0.0000 & 0.0000 & 0.0000 & 0.0000 & 0.0000 & 0.0000 & 0.0458 & 0.0048 & 0.0000 & 0.0000 & 0.0000 \\ \mathrm{Fe} & 8.0194 & 7.7937 & 7.9288 & 8.1207 & 7.9441 & 7.9949 & 7.9989 & 8.0340 & 7.9736 & 7.9144 & 80641 \\ \mathrm{Zn} & 0.0000 & 0.0000 & 0.0000 & 0.0000 & 0.0000 & 0.0000 & 0.0000 & 0.0200 & 0.0000 & 0.0254 & 0.0000 \\ \mathrm{Mn} & 0.0000 & 0.0158 & 0.0161 & 0.0202 & 0.0180 & 0.0101 & 0.0102 & 0.0000 & 0.0027 & 0.0185 & 0.0105 \\ \mathrm{Ca} & 0.0034 & 0.0167 & 0.0000 & 0.0640 & 0.0000 & 0.0000 & 0.0000 & 0.0065 & 0.0000 & 0.0101 & 0.0000 \\ \mathrm{~K} & 0.0000 & 0.0000 & 0.0000 & 0.0114 & 0.0000 & 0.0000 & 0.0000 & 0.0000 & 0.0000 & 0.0000 & 0.0040 \\ \mathrm{Na} & 0.0122 & 0.0181 & 0.0000 & 0.0058 & 0.0000 & 0.0000 & 0.0116 & 0.0000 & 0.0000 & 0.0243 & 0.0000\end{array}$

Host rock, occurrence $(\mathrm{Kj}=$ Kortejärvi, $\mathrm{Lj}=$ Laivajoki), drill hole/depth $(\mathrm{m})$ :

1 = dolomite carbonatite

2 = olivine-magnetite rock

3 = olivine-magnetite rock

4 = olivine-magnetite rock

$5=$ olivine-magnetite rock

6 = calcite carbonatite
Kj 118.40

Kj 143.00

Kj 137.00

Kj 252.70

$\mathrm{Kj} 252.70$

Lj 560.25
7 = tremolite-dolomite carbonatite

8 = tremolite-dolomite carbonatite

9 = tremolite-dolomite carbonatite

$10=$ serpentine-dolomite carbonatite

$11=$ serpentine-dolomite carbonatite
Lj 35.60

Lj 375.60

Lj 550.85

Lj 644.40

Lj 644.40
Allanite-(Ce) (EDS identification) is evenly distributed in both intrusions, especially in the c-carbonatites and the Laivajärvi tremolite-c-carbonatite. It is associated with amphiboles, phlogopite and magnetite. The Laivajärvi allanite displays irregular zoning of iron and is enriched in LREEs. The cracks in the allanite are filled with a lanthanide-rich mineral, the chemical composition of which indicates that it could be cerite-(Ce) (cf. Burt 1989).

Allanite is a common mineral in carbonatites (Hogarth 1989), and Mariano (1989b) considers it a hydrothermal product which occurs as a vein fill or an alteration product of primary minerals. The Kortejärvi and Laivajärvi allanite does not occur in veins, but as solitary grains. 
Table 7. Chemical compositions of ilmenites of the Kortejärvi (Nos. 1-5) and Laivajoki (Nos. 6-8) carbonatites, Finland. Analyst. J. Nykänen.

\begin{tabular}{lrrrrrrrr} 
& \multicolumn{1}{c}{1} & \multicolumn{1}{c}{2} & \multicolumn{1}{c}{3} & \multicolumn{1}{c}{4} & \multicolumn{1}{c}{5} & \multicolumn{1}{c}{6} & \multicolumn{1}{c}{7} & \multicolumn{1}{c}{8} \\
\hline $\mathrm{SiO}_{2}$ & 0.01 & 0.02 & 0.03 & 0.01 & 0.01 & 0.03 & 0.00 & 0.02 \\
$\mathrm{TiO}_{2}$ & 51.38 & 53.28 & 51.88 & 53.56 & 54.51 & 50.62 & 53.15 & 52.70 \\
$\mathrm{Al}_{2} \mathrm{O}_{3}$ & 0.00 & 0.00 & 0.00 & 0.01 & 0.00 & 0.01 & 0.01 & 0.00 \\
$\mathrm{Cr}_{2} \mathrm{O}_{3}$ & 0.02 & 0.03 & 0.00 & 0.00 & 0.05 & 0.03 & 0.00 & 0.00 \\
$\mathrm{~V}_{2} \mathrm{O}_{3}$ & 0.00 & 0.00 & 0.00 & 0.00 & 0.00 & 0.00 & 0.00 & 0.00 \\
$\mathrm{Fe}_{2} \mathrm{O}_{3}$ & 0.47 & 0.41 & 0.80 & 0.07 & 0.16 & 1.14 & 0.35 & 0.14 \\
$\mathrm{FeO}$ & 45.12 & 33.28 & 33.09 & 37.79 & 39.62 & 44.07 & 43.49 & 40.11 \\
$\mathrm{MnO}$ & 0.81 & 2.11 & 2.17 & 2.17 & 2.04 & 1.24 & 2.07 & 1.81 \\
$\mathrm{MgO}$ & 0.28 & 7.13 & 6.60 & 4.61 & 4.14 & 0.40 & 1.27 & 3.04 \\
$\mathrm{ZnO}$ & 0.00 & 0.00 & 0.00 & 0.00 & 0.07 & 0.06 & 0.13 & 0.13 \\
$\mathrm{NiO}$ & 0.00 & 0.00 & 0.02 & 0.00 & 0.00 & 0.00 & 0.02 & 0.06 \\
$\mathrm{CaO}$ & 0.00 & 0.01 & 0.00 & 0.01 & 0.00 & 0.00 & 0.00 & 0.00 \\
$\mathrm{Na} 2 \mathrm{O}$ & 0.00 & 0.01 & 0.00 & 0.00 & 0.01 & 0.00 & 0.00 & 0.00 \\
$\mathrm{~K}_{2} \mathrm{O}$ & 0.00 & 0.00 & 000 & 0.00 & 0.01 & 0.02 & 0.01 & 0.01 \\
\hline $\mathrm{Total}$ & 98.08 & 96.27 & 94.59 & 98.23 & 100.63 & 97.62 & 100.50 & 98.03
\end{tabular}

Cations per 6 oxygen atoms

$\begin{array}{lllllllll}\mathrm{Si} & 0.0005 & 0.0010 & 0.0015 & 0.0005 & 0.0005 & 0.0016 & 0.0000 & 0.0010 \\ \mathrm{Al} & 0.0000 & 0.0000 & 0.0000 & 0.0006 & 0.0000 & 0.0006 & 0.0006 & 0.0000 \\ \mathrm{Cr} & 0.0008 & \mathrm{n} .0 \mathrm{n} 12 & 0.0000 & 0.0000 & 0.0019 & 0.0012 & 0.0000 & 0.0000 \\ \mathrm{Fe} 3 & 0.0180 & 0.0152 & 0.0307 & 0.0027 & 0.0060 & 0.0444 & 0.0132 & 0.0054 \\ \mathrm{Ti} & 1.9869 & 1.9879 & 1.9778 & 1.9973 & 1.9940 & 1.9674 & 1.9905 & 1.9944 \\ \mathrm{~V} & 0.0000 & 0.0000 & 0.0000 & 0.0000 & 0.0000 & 0.0000 & 0.0000 & 0.0000 \\ \mathrm{Mg} & 0.0215 & 0.5273 & 0.4987 & 0.3407 & 0.3001 & 0.0308 & 0.0943 & 0.2280 \\ \mathrm{Ni} & 0.0000 & 0.0000 & 0.0008 & 0.0000 & 0.0000 & 0.0000 & 0.0008 & 0.0024 \\ \mathrm{Fe} & 1.9401 & 1.3806 & 1.4028 & 1.5671 & 1.6117 & 1.9047 & 1.8110 & 1.6882 \\ \mathrm{Zn} & 0.0000 & 0.0000 & 0.0000 & 0.0000 & 0.0025 & 0.0023 & 0.0048 & 0.0048 \\ \mathrm{Mn} & 0.0353 & 0.0887 & 0.0932 & 0.0911 & 0.0840 & 0.0543 & 0.0873 & 0.0771 \\ \mathrm{Ca} & 0.0000 & 0.000 \mathrm{~S} & 0.0000 & 0.0005 & 0.0000 & 0.0000 & 0.0000 & 0.0000 \\ \mathrm{Na} & 0.0000 & 0.0010 & 0.0000 & 0.0000 & 0.0009 & 0.0000 & 0.0000 & 0.0000 \\ \mathrm{~K} & 0.0000 & 0.0000 & 0.0000 & 0.0000 & 0.0006 & 0.0013 & 0.0006 & 0.0006\end{array}$

Host rock, occurrence $(\mathrm{Kj}=$ Kortejärvi, $\mathrm{Lj}=$ Laivajoki), drill hole/depth $(\mathrm{m})$ :

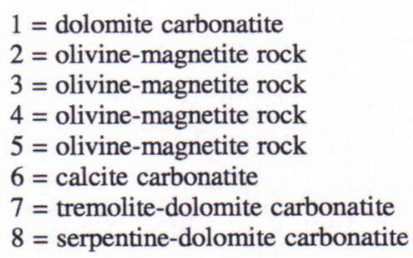

3 = olivine-magnetite rock

4 = olivine-magnetite rock

5 = olivine-magnetite rock

$6=$ calcite carbonatite

7 = tremolite-dolomite carbonatite

$8=$ serpentine-dolomite carbonatite

Kj 118.40 (grain)

Kj 137.00 (lamella)

Kj 137.00 (grain)

$\mathrm{Kj} 143.00$ (inner composite grain)

Kj 252.70 (marginal composite grain)

Lj 560.25 (grain)

Lj 375.60 (grain)

Lj 644.40 (grain) 


\section{WHOLE ROCK GEOCHEMISTRY OF THE KORTEJÄRVI AND LAIVAJOKI ROCKS}

It should be noted that all the samples analysed were split halves of drill cores $2.5 \mathrm{~cm}$ in diameter. They were fresh, but only about 5 to $10 \mathrm{~cm}$ long due to the scarcity of the material.

Table 8 gives main element analyses of the Kortejärvi and Laivajärvi rocks. In the main element classification of Woolley and Kempe (1989), the Kortejärvi d-carbonatites and c-d- carbonatites are magnesiocarbonatites, whereas, due their high magnetite content, the Kortejärvi and Laivajärvi c-carbonatites and one Kortejärvi d-c-carbonatite belong to the ferrocarbonatite group (Fig. 8). It is important to notice that the $\mathrm{CaO}$ contents of the carbonatites proper of both Kortejärvi and Laivajoki fill the $35-45 \% \mathrm{CaO}$ gap of Woolley and Kempe (1989, Fig. 1.1, for discussion of this enigma see Bailey 1993, p. 644).

Table 8. Chemical compositions of the main elements (wt-\%) of the rocks of the Kortejärvi (Nos. 1-7) and Laivajoki (Nos. 8-15) carbonatites, Finland. XRF-analyses, except FeO by titrimetry.

\begin{tabular}{|c|c|c|c|c|c|c|c|c|c|c|c|c|c|c|c|}
\hline & 1 & 2 & 3 & 4 & 5 & 6 & 7 & 8 & 9 & 10 & 11 & 12 & 13 & 14 & 15 \\
\hline $\mathrm{SiO}_{2}$ & 1.76 & 2.51 & 2.84 & 2.27 & 1.90 & 7.83 & 39.5 & 3.94 & 9.62 & 6.73 & 5.79 & 3.12 & 29.3 & 16.6 & 31.2 \\
\hline $\mathrm{TiO}_{2}$ & 0.26 & 0.22 & 0.08 & 0.17 & 0.11 & 2.26 & 0.33 & 0.38 & 0.32 & 0.43 & 0.73 & 0.32 & 4.71 & 3.78 & 0.36 \\
\hline $\mathrm{A}_{2} \mathrm{O}_{3}$ & 0.13 & 0.12 & 0.08 & 0.18 & 0.10 & 0.27 & 7.74 & 0.25 & 1.47 & 0.41 & 0.62 & 0.06 & 1.72 & 0.53 & 0.29 \\
\hline $\mathrm{Fe}_{2} \mathrm{O}_{3}$ & 5.52 & 5.13 & 0.87 & 4.72 & 1.95 & 46.28 & 1.76 & 3.69 & 1.25 & 3.48 & 6.49 & 1.22 & $38.4 *$ & 19.3 & 9.63 \\
\hline $\mathrm{FeO}$ & 3.99 & 4.47 & 4.46 & 4.17 & 4.57 & 22.42 & 6.89 & 3.39 & 3.87 & 4.35 & 4.69 & 3.63 & n.d. & 12.78 & 8.07 \\
\hline $\mathrm{MnO}$ & 0.21 & 0.19 & 0.27 & 0.20 & 0.23 & 0.23 & 0.12 & 0.29 & 0.18 & 0.27 & 0.19 & 0.40 & 0.50 & 0.53 & 0.27 \\
\hline $\mathrm{MgO}$ & 6.29 & 8.30 & 14.5 & 14.9 & 12.5 & 7.72 & 17.8 & 6.14 & 4.88 & 5.27 & 6.90 & 9.10 & 8.59 & 13.6 & 24.9 \\
\hline $\mathrm{CaO}$ & 41.9 & 38.2 & 32.2 & 31.4 & 34.9 & 4.18 & 9.76 & 42.2 & 40.5 & 41.8 & 37.7 & 39.6 & 9.16 & 12.6 & 9.10 \\
\hline $\mathrm{Na}_{2} \mathrm{O}$ & -- & 0.07 & 0.07 & 0.10 & 0.07 & 0.12 & 0.56 & 0.34 & 0.09 & -- & 0.10 & 0.14 & 0.14 & 0.12 & \\
\hline $\mathrm{K}_{2} \mathrm{O}$ & 0.17 & 0.17 & - & 0.27 & 0.08 & 0.13 & 5.84 & 0.19 & 0.98 & 0.25 & -- & 0.09 & 0.84 & 0.07 & 0.09 \\
\hline $\mathrm{P}_{2} \mathrm{O}_{5}$ & 0.14 & -- & 6.89 & 7.34 & 5.95 & 0.21 & 2.06 & 0.31 & 0.23 & 6.94 & 0.31 & 1.90 & -- & -- & -- \\
\hline LOI & 38.4 & 37.7 & 36.8 & 33.3 & 36.4 & 4.23 & 4.39 & 37.2 & 33.5 & 28.6 & 33.1 & 38.5 & -0.92 & 12.8 & 14.8 \\
\hline Total & 99.5 & 98.0 & 99.9 & 99.8 & 99.6 & 98.7 & 97.7 & 98.7 & 98.0 & 99.4 & -- & 98.9 & 92.7 & 94.4 & 100.2 \\
\hline
\end{tabular}

* total iron as $\mathrm{Fe}_{2} \mathrm{O}_{3}$. n.d. $=$ not determined

Rock, occurrence $(\mathrm{Kj}=$ Kortejärvi. $\mathrm{Lj}=$ Laivajoki $)$, drill hole/depth (m).

1 = calcite carbonatite $\mathrm{Kj} 156.20$

2 = dolomite-calcite carbonatite $\mathrm{Kj} 245.00$

3 = dolomite carbonatite $\mathrm{Kj} 118.40$

4 = dolomite carbonatite $\mathrm{Kj} 179.40$

5 = calcite-dolomite carbonatite $\mathrm{Kj} 273.95$

6 = olivine-magnetite rock $\mathrm{Kj} 143.00$

7 = glimmerite $\mathrm{Kj} 1110.25$

\author{
8 = calcite carbonatite $\mathrm{Lj} 383.50$ \\ $9=$ calcite carbonatite $\mathrm{Lj} 545.05$ \\ $10=$ calcite carbonatite Lj 560.25 \\ 11 = calcite carbonatite $\mathrm{Lj} 638.25$ \\ 12 = dolomite-calcite carbonatite $\operatorname{Lj} 647.40$ \\ 13 = tremolite-rich carbonatite $\mathrm{Lj} 371.20$ \\ 14 = tremolite-rich carbonatite $\mathrm{Lj} 539.80$ \\ $15=$ serpentine-talc-dolomite rock $\mathrm{Lj} 644.40$
}

The higher silicate content of the Laivajärvi carbonatites sensu stricto (modal carbonate content $>50 \%, \mathrm{SiO}_{2}<10 \%$ ) means that they are markedly richer in silica, $\mathrm{TiO}_{2}$ and $\mathrm{Al}_{2} \mathrm{O}_{3}$ than those at Kortejärvi (Table 8). Most of the Laivajärvi c-carbonatites have a $\mathrm{FeO}: \mathrm{Fe}_{2} \mathrm{O}_{3}$ ration $>1$, which indicates the presence of Fe-dolomite or ankerite, whereas, due to their more abundant magnetite, the ratio is reversed in the Kortejärvi carbonatites. The $\mathrm{P}_{2} \mathrm{O}_{5}$ content varies in both occurrences due to the uneven distribution of apatite, the content of which is $7-6 \%$ in the c-carbonatites or d-carbonatites at its highest. 


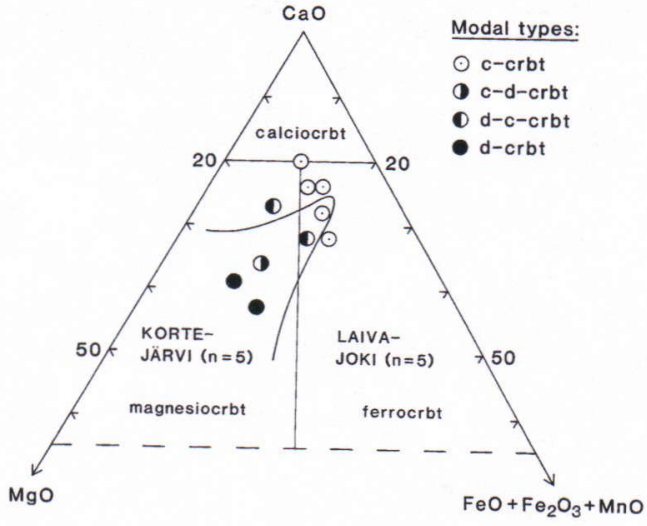

Fig. 8. Main element compositions of the Kortejärvi and Laivajoki carbonatites (carbonate and $\mathrm{SiO}_{2}$ contents $>50 \%$ and $<10 \%$, respectively) plotted on the Woolley's and Kempe's (1989) diagram. The location of the modally defined calcite-carbonatites within the ferrocarbonatite field is due to their magnetite contents.

\section{TRACE ELEMENT GEOCHEMISTRY OF THE KORTEJÄRVI AND LAIVAJO- KI ROCKS}

Trace element analyses are given in Tables 9 and 10. An interesting feature is low niobium content (9-30 ppm in carbonate carbonatites and c. 580 in tremolite-rich varieties) in carbonatites, although enrichment of this element is commonly considered typical to carbonatites (Woolley and Kempe 1989) and a good device to discriminate sedimentary carbonate rocks from carbonatites (Heinrich 1966). Samoilov (1991) states that $\mathrm{Nb}$ content varies greatly from an intrusion to another. Bowden $(1962,1968)$, Barber (1974), Hogarth et al. (1985) and Mariano (1989a) have reported $\mathrm{Nb}$-poor carbonatites from East Africa and Canada. Barber (1974) concludes that $\mathrm{Nb}$ is not so good a carbonatite indicator than REEs. Also Bedard and Chown (1992) agree that a rock can be a carbonatite in spite of low $\mathrm{Nb}$ content. Möller (1989a) states that the $\mathrm{Nb}(\mathrm{Ta})$ amounts in carbonatites and alkaline rocks depend on the partial melting of metasomatically altered mantle.
Table 9. Trace element contents (ppm., methods: $\mathrm{Sc}, \mathrm{Co}, \mathrm{Sr}, \mathrm{Y}, \mathrm{Zr} / \mathrm{ICP} ; \mathrm{V}, \mathrm{Cr}, \mathrm{Ni}, \mathrm{Cu}, \mathrm{Zn} / \mathrm{DCP} ; \mathrm{Rb}$, $\mathrm{Nb} / \mathrm{XRF}$; Cs, Hf, Ta, Tl, u/NA; REEs/ICP-MS) of the rocks of the Kortejärvi carbonatite, Finland.

\begin{tabular}{lrrrrrrr} 
& 1 & 2 & 3 & 4 & 5 & 6 & 7 \\
\hline $\mathrm{Sr}$ & 2510 & 2090 & 2560 & 1870 & 2200 & 122 & 548 \\
$\mathrm{Ba}$ & 107 & 198 & 79 & 31 & 84 & 137 & 763 \\
$\mathrm{Sc}$ & 18 & 20 & 12 & 9 & 12 & 9 & 15 \\
$\mathrm{~V}$ & 100 & 160 & 50 & 120 & 74 & 1100 & 72 \\
$\mathrm{Cr}$ & $<2$ & $<2$ & $<2$ & $<2$ & $<2$ & $<2$ & 88 \\
$\mathrm{Co}$ & 26 & 31 & 12 & 24 & 27 & 118 & 37 \\
$\mathrm{Ni}$ & 6 & 2 & 3 & 4 & 3 & 2 & 32 \\
$\mathrm{Cu}$ & 4.0 & 4.5 & 12.0 & $<0.5$ & 11.0 & 3.5 & $<0.5$ \\
$\mathrm{Zn}$ & 51.0 & 36.0 & 29.0 & 47.0 & 32.0 & 250 & 96.0 \\
$\mathrm{Rb}$ & 15 & 11 & 11 & 21 & 8 & 6 & 249 \\
$\mathrm{Y}$ & 62 & 52 & 37 & 35 & 41 & 4 & 21 \\
$\mathrm{Zr}$ & 10 & 976 & 95 & 633 & 236 & 1620 & 66 \\
$\mathrm{Nb}$ & 16 & 16 & 8 & 15 & 9 & 161 & 91 \\
$\mathrm{Cs}$ & $<1$ & $<1$ & $<1$ & $<1$ & $<1$ & $<1$ & 6 \\
$\mathrm{Hf}$ & $<1$ & 18 & 2 & 9 & 4 & 28 & 1 \\
$\mathrm{Ta}$ & 3 & 3 & $<1$ & 2 & 1 & 14 & 4 \\
$\mathrm{~Pb}$ & $<2$ & $<2$ & $<2$ & 4 & $<2$ & $<2$ & $<2$ \\
$\mathrm{Th}$ & $<0.5$ & $<0.5$ & 1.1 & 1.4 & 0.9 & 0.7 & 3.4 \\
$\mathrm{U}$ & $<0.1$ & 0.1 & 0.4 & 0.3 & 0.3 & 0.4 & 0.5 \\
$\mathrm{La}$ & 158 & 134 & 142 & 132 & 135 & 12.5 & 75.4 \\
$\mathrm{Ce}$ & 314 & 265 & 320 & 297 & 292 & 30.1 & 165 \\
$\mathrm{Pr}$ & 42.2 & 35.4 & 43.6 & 43.7 & 41.1 & 3.9 & 20.9 \\
$\mathrm{Nd}$ & 187 & 157 & 203 & 199 & 183 & 16.8 & 89.7 \\
$\mathrm{Sm}$ & 34.6 & 29.2 & 34.2 & 34.3 & 33.1 & 2.6 & 15.6 \\
$\mathrm{Eu}$ & 10.5 & 8.95 & 9.44 & 9.99 & 9.80 & 0.85 & 4.15 \\
$\mathrm{Gd}$ & 30.1 & 25.5 & 25.7 & 26.6 & 27.9 & 2.1 & 12.4 \\
$\mathrm{~Tb}$ & 3.6 & 3.2 & 2.7 & 3.0 & 3.2 & 0.3 & 1.4 \\
$\mathrm{Dy}$ & 18.0 & 15.0 & 12.4 & 13.5 & 14.5 & 1.2 & 6.6 \\
$\mathrm{Ho}$ & 2.96 & 2.49 & 1.79 & 1.93 & 2.07 & 0.17 & 0.98 \\
$\mathrm{Er}$ & 6.4 & 5.2 & 3.3 & 3.6 & 4.3 & 0.5 & 2.2 \\
$\mathrm{Tm}$ & 0.7 & 0.6 & 0.3 & 0.3 & 0.5 & $<0.1$ & 0.2 \\
$\mathrm{Yb}$ & 4.0 & 3.3 & 1.8 & 1.6 & 2.5 & 0.2 & 1.2 \\
$\mathrm{Lu}$ & 0.48 & 0.37 & 0.13 & 0.16 & 0.30 & $<0.05$ & 0.15 \\
\hline $\mathrm{REE}(\mathrm{Y}) \mathrm{rot}$ & 874.54 & 737.21 & 837.36 & 801.68 & 790.27 & 75.22 & 416.88 \\
& & & & & & &
\end{tabular}

Rock, drill hole/depth (m).

$1=$ calcite carbonatite 156.20

$2=$ dolomite-calcite carbonatite 245.00

$3=$ dolomite carbonatite 118.40

4= dolomite carbonatite 179.40

$5=$ calcite-dolomite carbonatite 273.95

$6=$ olivine-magnetite rock 143.00

$7=$ glimmerite 1110.25

$\mathrm{Nb}$ in carbonatites is most commonly carried by pyrochlore (Le Bas 1977, Kapustin 1980, Woolley and Kempe 1989). Due to the low Nb contents this as well as other $\mathrm{Nb}$-minerals (e.g. fersmite, columbite, perovskite) are not encountered in the Kortejärvi and Laivajärvi rocks. The Kortejärvi olivine-magnetite rock and glimmerite and the Laivajärvi tremolite-rich carbonatites contain markedly more $\mathrm{Nb}$ than other rocks. In these rock $\mathrm{Nb}$ is evidently hosted by magnetite, phlogopite and tremolite (cf. Morteani 1989). 
Table 10. Trace element contents (ppm. XRF analyses for methods see Table 9) of the Laivajoki rocks.

\begin{tabular}{|c|c|c|c|c|c|c|c|c|}
\hline & 1 & 2 & 3 & 4 & 5 & 6 & 7 & 8 \\
\hline $\mathrm{Sr}$ & 2890 & 2920 & 2370 & 1830 & 3480 & 198 & 456 & 505 \\
\hline $\mathrm{Ba}$ & 84 & 348 & 85 & 27 & 85 & 198 & 43 & 45 \\
\hline $\mathrm{Sc}$ & 11 & 14 & 47 & 8 & 29 & 94 & 6 & 17 \\
\hline V & 160 & 88 & 300 & 190 & 30 & 1300 & 600 & 150 \\
\hline $\mathrm{Cr}$ & $<2$ & $<2$ & 68 & $<2$ & $<2$ & $<2$ & $<2$ & $<2$ \\
\hline Co & 31 & 24 & 26 & 35 & 21 & 114 & 59 & 68 \\
\hline $\mathrm{Ni}$ & 12 & $<1$ & 21 & 6 & 10 & 25 & 8 & 5 \\
\hline $\mathrm{Cu}$ & 53.0 & 50.0 & 140 & 46.0 & 42.0 & 660 & 120 & 140 \\
\hline $\mathrm{Zn}$ & 30.0 & 42.0 & 27.0 & 26.0 & 25.0 & 150 & 86.0 & 88.0 \\
\hline $\mathrm{Rb}$ & 13 & 41 & 12 & 8 & 8 & 27 & 7 & 8 \\
\hline $\mathrm{Y}$ & 83 & 83 & 88 & 69 & 74 & 7 & 22 & 10 \\
\hline $\mathrm{Zr}$ & 169 & 513 & 97 & 437 & 133 & 1490 & 1090 & 2860 \\
\hline $\mathrm{Nb}$ & 13 & 29 & 16 & 13 & 30 & 589 & 573 & 10 \\
\hline Cs & 1 & $<1$ & $<1$ & $<1$ & $<1$ & $<1$ & 1 & $<1$ \\
\hline Hf & 5 & 16 & 4 & 13 & 6 & 54 & 28 & 64 \\
\hline $\mathrm{Ta}$ & 1 & 2 & 2 & 3 & 5 & 11 & 7 & $<1$ \\
\hline $\mathrm{Pb}$ & $<2$ & $<2$ & $<2$ & $<2$ & $<2$ & $<2$ & $<2$ & $<2$ \\
\hline Th & 0.8 & 2.4 & 0.9 & 1.6 & 2.4 & 0.8 & 0.6 & 0.5 \\
\hline $\mathrm{U}$ & $<0.1$ & 0.4 & 0.7 & 0.4 & 0.9 & 0.2 & 0.1 & $<0.1$ \\
\hline $\mathrm{La}$ & 228 & 239 & 244 & 261 & 171 & 11.7 & 66.1 & 24.7 \\
\hline $\mathrm{Ce}$ & 393 & 439 & 537 & 493 & 363 & 24.8 & 121 & 47.8 \\
\hline $\operatorname{Pr}$ & 51.8 & 55.4 & 71.3 & 63.1 & 47.0 & 3.5 & 15.4 & 5.9 \\
\hline $\mathrm{Nd}$ & 225 & 240 & 323 & 267 & 208 & 17.7 & 68.6 & 25.5 \\
\hline $\mathrm{Sm}$ & 39.2 & 43.3 & 58.6 & 46.2 & 37.8 & 3.8 & 12.4 & 4.3 \\
\hline $\mathrm{Eu}$ & 10.5 & 13.4 & 17.1 & 12.6 & 11.2 & 1.88 & 2.94 & 1.42 \\
\hline $\mathrm{Gd}$ & 36.6 & 40.5 & 53.7 & 39.5 & 34.5 & 3.6 & 11.5 & 4.0 \\
\hline $\mathrm{Tb}$ & 4.6 & 4.8 & 6.6 & 4.5 & 4.3 & $0 . S$ & 1.5 & 0.5 \\
\hline Dy & 21.7 & 22.9 & 29.0 & 22.7 & 18.6 & 2.4 & 6.7 & 2.3 \\
\hline Ho & 3.68 & 3.82 & 4.68 & 3.77 & 3.28 & 0.34 & 1.06 & 0.36 \\
\hline $\mathrm{Er}$ & 8.4 & 8.5 & 10.2 & 8.3 & 7.0 & 0.8 & 2.5 & 0.7 \\
\hline $\mathrm{Tm}$ & 1.0 & 1.0 & 1.2 & 0.9 & 0.9 & 0.1 & 0.3 & 0.1 \\
\hline $\mathrm{Yb}$ & 5.7 & 5.9 & 6.7 & 5.4 & 5.5 & 0.6 & 1.4 & 0.5 \\
\hline $\mathrm{Lu}$ & 0.71 & 0.75 & 0.95 & 0.64 & 0.7 & 0.05 & 0.18 & 0.05 \\
\hline REE & & 1112.89 & 1201.27 & 1452.03 & 1297.61 & 986.78 & 78.77 & 333.58 \\
\hline
\end{tabular}

Rock, drill hole/depth (m)

1 = calcite carbonatite 383.50

2 = calcite carbonatite 545.05

$3=$ calcite carbonatite 560.25

$4=$ calcite carbonatite 638.25
$5=$ dolomite-calcite carbonatite 647.40
$6=$ tremolite-rich carbonatite 371.20
$7=$ tremolite-rich carbonatite 539.80
$8=$ serpentine-talc-dolomite rock 644.40
The contents of $U(<1 \mathrm{ppm})$ and Th $(<3.5$ ppm) in the Kortejärvi and Laivajärvi rocks are lower than the average contents given by Woolley and Kempe (1989). This is the cause that no perovskite, pyrochlore and thorite and only small amounts of REE-minerals are met with in them.

The Sr contents (122-3480 ppm) of the Kortejärvi and Laivajärvi rocks are typical, but not noticeably high to carbonatites and correlates well with the carbonate content (or LOI) of the rock.

Barium contents (31-348 ppm) of the carbonatites are rather low. It is highest in the Kortejärvi glimmerite (763 ppm) and the Laivajärvi phlogopite-bearing c-carbonatite (348 ppm), which indicates that $\mathrm{Ba}$ is located in phlogopite. 
The amount of $\mathrm{Zr}$ varies greatly being $\mathrm{n} \mathrm{x}$ $100 \mathrm{ppm}$ in carbonatites and in the Kortejärvi glimmerite, but is markedly higher in the Kortejärvi olivine-magnetite rock (1620 ppm, Table 9) and in the silicate-rich Laivajärvi rocks where it rises up to $2860 \mathrm{ppm}$ (Table 10).

Vanadium content is highest (up to $1100 \mathrm{ppm}$ ) in the magnetite-rich rocks.

\section{RARE EARTH ELEMENT GEOCHEM- ISTRY OF THE KORTEJÄRVI AND LAIVAJOKI ROCKS}

Carbonatites contain the highest amounts of REE and highest LREE/HREE ratios $(\mathrm{La} / \mathrm{Lu}=7.1$ -1240 ) of any rock type (Cullers and Graaf 1984). REE contents vary from 72 to 15515 ppm (op. cit.), but some carbonatite veins may contain up to 41000 ppm REE (Hogarth et al. 1985). High LREE contents are treated by numerous authors (Heinrich 1966, Loubet et al. 1972, Eby 1975, Mitchell and Brundfelt 1975, Hogarth et al. 1988, Nelson et al. 1988). When a variety of carbonatites is present, it is almost always rocks emplaced late in the sequence that contain the greatest abundance of REE. These "late" carbonatites are often the ferrocarbonatites (Wall and Mariano 1996).

The total REE contents of the Kortejärvi and Laivajärvi rocks are relatively low (Tables 9 and 10), but the LREE/HREE ratios are distinguishingly high in both occurrences (Figs 9 and 10). REE(Y) levels are markedly higher in Laivajoki (1113-1452 ppm in c-carbonatites and $987 \mathrm{ppm}$ in d-carbonatite) than in Kortejärvi (738-875 ppm in c-carbonatite and d-carbonatites). REE contents in the silicate-bearing rocks are much lower. All the chondrite normalised REE curves are steeply falling (Figs 9 and 10), but the Kortejärvi d-carbonatites are extremely impoverished in HREE.

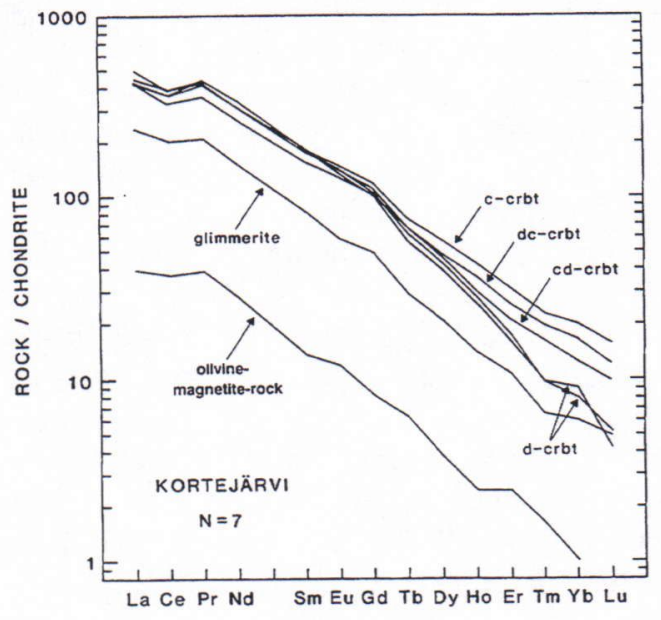

Fig. 9. Chondrite normalized REE distribution curves of the Kortejärvi rocks. Anal. ICP-MS, X-ray Assay Laboratories, Ontario.

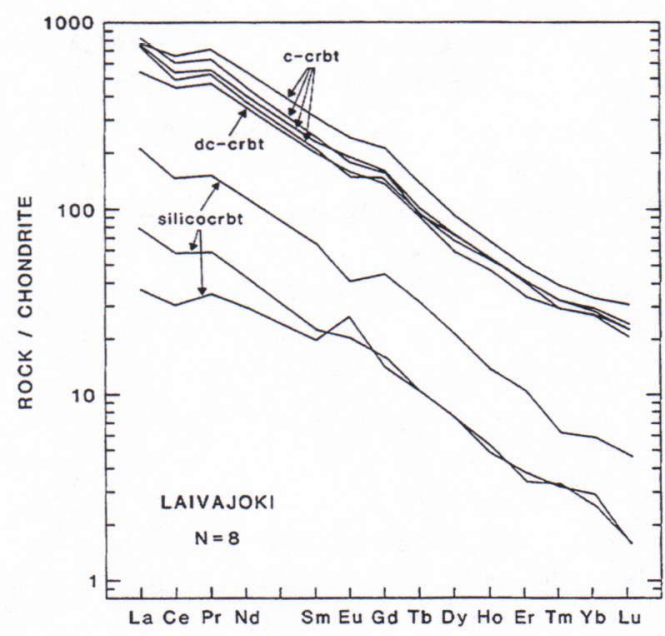

Fig. 10. Chondrite normalized REE distribution curves of the Laivajoki rocks. Anal. ICP-MS, X-ray Assay Laboratories, Ontario.

Calcite and apatite are main carriers of lanthanides in early stage carbonatites (Kapustin 1966, Cullers and Graf 1984, Möller 1989b). When dolomite and apatite replace calcite metasomatically lanthanides are released and mona- 
zite and fluorocarbonates are crystallised from fluids. Dolomite and ankerite do not contain lanthanides as much as calcite (Sokolov 1985). Because the Kortejärvi and Laivajärvi carbonates and apatites do not contain REE, the main lanthanide carriers are allanite and monazite.

The Laivajärvi tremolite carbonatites show either a positive or negative Eu anomaly. According to Möller (1989 a \& b) this feature can not be primary magmatic, but is due to later alteration.

All the Kortejärvi and Laivajärvi samples show negative $\mathrm{Ce}$ anomalies, which indicates that the magma was formed under oxidising conditions (Loube et al. 1972, Möller et al. 1980).

\section{CARBON AND OXYGEN ISOTOPE RA- TIOS OF THE KORTEJÄRVI AND LAI- VAJOKI ROCKS}

Analyses of coexisting calcite and dolomite were made using a modification of the method described by Epstein et al. (1964), which is based on the different reaction rates of calcite and dolomite with phosphoric acid. Analytical details followed the procedure given by Karhu (1993). Particle size ranges and reaction times were optimized in order to minimize cross-mixing of $\mathrm{CO}_{2}$ evolved from the two phases, and the resulting bias was expected to remain below about 0.1 for carbon isotopes and below about 0.2 for oxygen isotope ratios.

Carbon and oxygen analytical results of the carbonatite samples from the Laivajoki and Kortejärvi intrusions are given in Table 11 and illustrated in Figure 11a. In addition to the isotopic data, Table 11 also gives the proportion of dolomite in the total carbonate fraction as determined by XRD analysis. Analyzed samples represent relatively pure carbonate rocks, which according to acid reaction yields contain more than $80 \%$ carbonate. In the sample material no systematical differences can be observed between calcite and dolomite carbonatites or between various parts of the intrusions.

The mean $\delta^{13} \mathrm{C}$ values of dolomite and calcite for the Laivajoki samples are $-4.3 \pm 0.1 \%$ o $( \pm 1 \mathrm{sd}$, PDB) and $-4.5 \pm 0.6 \%$, respectively, and for the Kortejärvi samples $-3.9 \pm 0.2 \%$ o and $-4.2 \pm 0.2 \%$, respectively. Compared to carbon the isotope ratios of oxygen show more variation. The mean ${ }^{18} \mathrm{O}$ values of dolomite and calcite for the Laivajoki samples are $6.5 \pm 0.5 \%$ o $( \pm 1 \mathrm{sd}$, SMOW) and $7.0 \pm 0.8 \%$, respectively, and for the Kortejärvi intrusion $6.8 \pm 0.1 \%$ o and $8.4 \pm 1.0 \%$, respectively. These isotopic characteristics are similar to the analytical results reported from other carbonatite complexes, and, more specifically, they are in the range expected for carbonate melts in equilibrium with mantle minerals (Deines 1989).

The fractionation of carbon isotopes between coexisting dolomite and calcite in the Laivajoki and Kortejärvi intrusions is relatively invariable with $\Delta^{13} \mathrm{C}$ (dolomite-calcite) varying between 0.35 and 0.75 (Table 11, Fig. 11b). On the basis of an empirical fractionation calibration of Sheppard and Schwarcz (1970) these values are suggestive of preservation of a high temperature equilibrium relation between the two minerals.

In contrast to carbon, all oxygen isotope fractionations between coexisting dolomite and calcite are reversed. The $\Delta^{18} \mathrm{O}$ (dolomite-calcite) values are all negative and range from -0.4 to -2.5 (Fig. 11b), whereas at equilibrium dolomite may be expected to be more enriched in ${ }^{18} \mathrm{O}$ than calcite (Sheppard and Schwarcz 1970). Oxygen isotope disequilibrium between coexisting dolomite and calcite has also been observed in many other carbonatite complexes (Deines, 1989), and besides it seems to be a general feature of dolomite-calcite pairs in high grade marbles (Sheppard and Schwarcz 1970; Karhu 1993). 
Table 11. Carbon and oxygen isotope data for samples from the Laivajoki and Kortejärvi carbonatite intrusions, Finland. Analyst. Juha Karhu.

\begin{tabular}{|c|c|c|c|c|}
\hline $\begin{array}{l}\text { Sample } \\
\text { drillhole/ } \\
\text { depth (m) }\end{array}$ & $\begin{array}{l}\text { Dolomite }{ }^{\&} \\
\text { wt. \% }\end{array}$ & Mineral $^{\S}$ & $\begin{array}{l}\delta^{13} \mathrm{C}, \\
\mathrm{PDB}\end{array}$ & $\begin{array}{l}\delta^{18} \mathrm{O} \% \text {, } \\
\text { SMOW }\end{array}$ \\
\hline \multicolumn{5}{|l|}{ Laivajoki } \\
\hline $\mathrm{R}-6 / 36.0$ & 0 & $\mathrm{Cal}$ & -3.60 & 5.91 \\
\hline \multirow[t]{2}{*}{ R-6/36.8-52.7 } & 26 & $\mathrm{Cal}$ & -4.78 & 7.44 \\
\hline & & Dol & -4.34 & 7.08 \\
\hline \multirow{2}{*}{ R-6/54.0 } & 35 & Cal & -4.79 & 7.38 \\
\hline & & Dol & -4.45 & 6.02 \\
\hline \multirow[t]{2}{*}{ R-6/55.8 } & 22 & $\mathrm{Cal}$ & -4.92 & 7.41 \\
\hline & & Dol & -4.17 & 6.44 \\
\hline \multicolumn{5}{|l|}{ Kortejärvi } \\
\hline $\mathrm{R}-1 / 18.5$ & 94 & Dol & -4.17 & 6.58 \\
\hline \multirow[t]{2}{*}{$\mathrm{R}-1 / 63.0$} & 35 & Cal & -4.33 & 9.30 \\
\hline & & Dol & -3.86 & 6.85 \\
\hline \multirow[t]{2}{*}{$\mathrm{R}-2 / 51.0$} & 55 & Cal & -4.04 & 7.48 \\
\hline & & Dol & -3.67 & 6.82 \\
\hline \multirow[t]{2}{*}{ R-2/68.5 } & 26 & Cal & -4.30 & 8.49 \\
\hline & & Dol & -3.86 & 6.90 \\
\hline
\end{tabular}

\&) Weight- $\%$ dolomite in total carbonate fraction by XRD,

§) $\mathrm{Cal}=$ Calcite, $\mathrm{Dol}=$ Dolomite
The mineral assemblages of the Laivajoki and Kortejärvi intrusions have experienced metamorphic high grade conditions during the Svecokarelian orogeny, and the isotope signatures of the calcite-dolomite pairs have certainly been reequilibrated at that time. Thus, the distinct oxygen isotope disequilibrium observed between calcite and dolomite apparently reflects processes that operated during post-metamorphic cooling of the region.

Reversed mineral-mineral fractionations are generally regarded as resulting from open-system conditions, in which one of the minerals exchanges with an infiltrating fluid at a higher rate than the others (Gregory and Criss 1986). Nevertheless, isotopic reversals may also arise from retrograde exhange reactions in closed-system conditions (e.g., Jenkin et al. 1991), which possibility is discussed in the following.
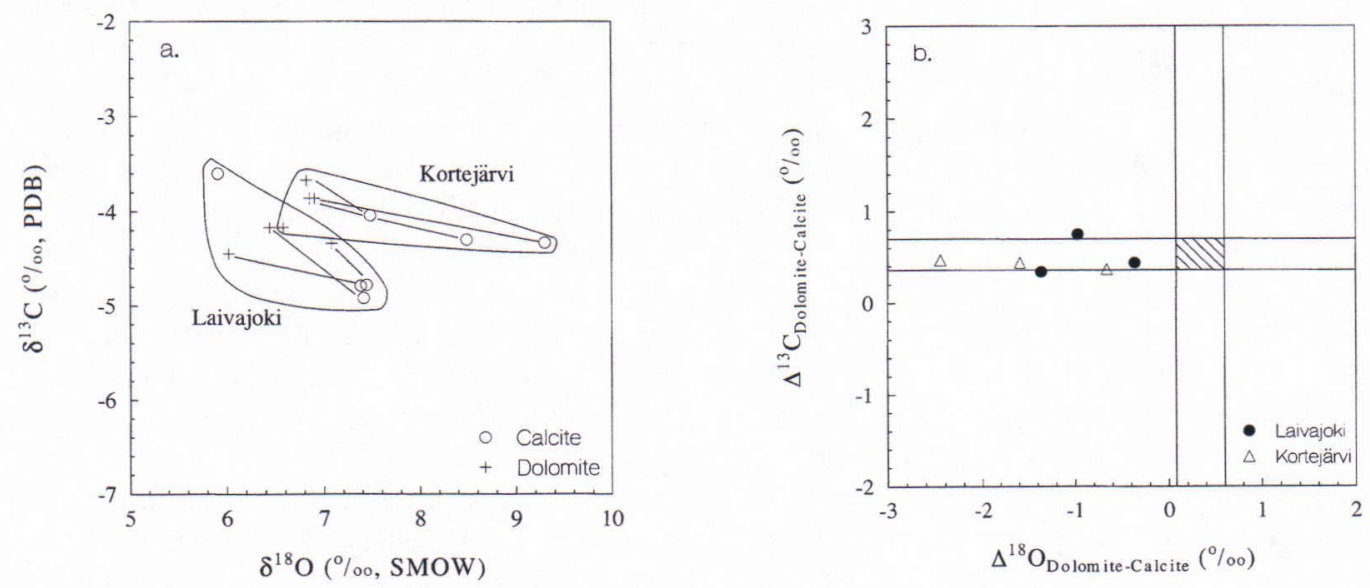

Fig. 11. Stable isotope systematics of carbonate samples from the Laivajoki and Kortejärvi intrusions. A) Relationship between the $\delta^{13} C$ and $\delta^{18} O$ values for calcite and dolomite. Tie lines combine results from coexisting mineral pairs. $B) \Delta^{13} C$ vs. $\Delta^{18} O$ plot for the dolomite-calcite system. The narrow fields define areas, where dolomite and calcite are in equilibrium at $400-700^{\circ} \mathrm{C}$ with respect to isotopic composition of carbon or oxygen. The crosshatched rectangle would include dolomite-calcite pairs at high temperature equilibrium relative both to carbon and oxygen isotope ratios. Fractionation calibrations are based on Sheppard and Schwarcz (1970). 
Among the two carbonate species present in the Laivajoki and Kortejärvi carbonatites, calcite is more susceptible to isotopic exchange than dolomite (Northrop and Clayton 1966), and during slow cooling dolomite may therefore be expected to be closed to oxygen isotope exchange before calcite. After dolomite is closed, calcite may continue to equilibrate with other carbonatite minerals like phlogopite, which is also known to be susceptible to low temperature exchange. At equilibrium calcite concentrates ${ }^{18} \mathrm{O}$ relative to most silicate and oxide phases present in carbonatites, and, in addition, these fractionations increase with decreasing temperatures (e.g., Kyser 1987). As a result of exchange, the $\delta^{18} \mathrm{O}$ values of calcite increase so that they may eventually exceed those of dolomite. In contrast to oxygen, the carbon isotope composition of calcite will remain unchanged after closing of dolomite, as these two are the only carbon bearing phases in carbonatites.

Accordingly, retrograde exhange reactions in closed-system conditions appear to be sufficient to explain both the preservation of the high temperature equilibrium for carbon and the distinct disequilibrium for oxygen characterizing the carbonatite samples from the Laivajoki and Kortejärvi inrusions. However, more data would be needed to fully constrain the model and to exclude the possibility of infiltration and exchange with external hydrous fluids.

\section{DISCUSSION}

Both the mineralogical and geochemical data, especially the REE analyses and carbon and oxygen isotope results, treated above prove that the Kortejärvi and Laivajärvi occurrences are true carbonatite intrusions. The differences in their petrography and geochemistry, again especially as to the REE contents, suggest that these bodies do not represent one single carbonatite body fragmented by the Svecokarelian orogeny, but two primarily separate carbonatites. The carbonatites may have, however, been evolved from a common parental magma. In this case, the more magnesiocarbonatitic (Fig. 8) and a little REE-poorer Kortejärvi body may represent the earlier phase.

Because the Kortejärvi and Laivajärvi occurrences are completely covered by surficial deposits and are intensely deformed and the number of drill holes is limited the mutual relations and proportions of their diverse rock types can not be established. Consequently, this discussion of their petrogenesis relies on literature.

Kapustin (1966) and Sokolov (1985) have discussed the subdivision of the Russian carbonatites into stages. On the basis of their mineralogy (calcite, forsterite, phlogopite, magnetite, apatite), both the Kortejärvi and Laivajärvi occurrences represent Kapustin's early stage carbonatites, but are somewhat altered as proved by the presence of clinohumite, dolomite, actinolite-tremolite, ilmenite, pyrrhotite and zircon. The Laivajärvi occurrence is more altered than the Kortejärvi one, because it does not contain fresh olivine, but contains Fe-dolomite and ankerite and is richer in tremolite, actinolite, zircon and ilmenite.

Sokolov (1985) takes into account both mineralogy and the mineral chemistry of carbonates. In this classification, the Kortejärvi olivine-magnetite rock represents II-III stages, because it contains relatively $\mathrm{Fe}$-poor dolomite in addition to olivine, clinohumite and calcite, whereas the c-carbonatite and d-carbonatite belong to stages III-IV because they contain Fe-dolomite, dolomite and even siderite. Th Laivajärvi rocks contain abundant amphibole and serpentine, but not olivine and their carbonates are Fe-dolomite and ankerite, which all define them into Sokolov's IV-V stages.

Gaspar (1992) considers clinohumite as metasomatic. An additional evidence of this process is that the Kortejärvi and Laivajärvi apatites and calcites do not contain REEs, but these elements are encountered in monazite and allanite.

If the $2020 \mathrm{Ma} \mathrm{U}-\mathrm{Pb}$ age of the Laivajärvi zircon (Vartiainen and Woolley 1974) dates the primary crystallization event, the intrusion of the Laivajärvi and Kortejärvi may be an early forerunner of the c. $1970 \mathrm{Ma}$ rifting of the Karelian 
craton. The $1875 \mathrm{Ma} \mathrm{K}-\mathrm{Ar}$ age of the Kortejärvi phologopite (Kresten et al. 1977) represents quite likely resetting of the K-Ar systematics during the Svecokarelian metamorphism.

ACKNOWLEDGEMENTS: This study is a contribution of IGCP 314 "Alkaline and carbonatitic magmatism" and the research projects "Metamorphism and Deformation of the Crust" (active
1988 - 1993) and "Mineral Resources and Norther Wellbeing ", supported by the Academy of Finland and the Thule Institute, University of Oulu. Lapin Malmi Oy kindly gave access to their archives. Alexander Korobeinikov and Seppo Gehör helped in some mineral calculations and presentations. Meri-Liisa Airo compiled the aeromagnetic map of Fig. 2. The English of the manuscript was checked by Dr. Malcom Hicks. 


\section{REFERENCES}

Barber, C. (1974) The geochemistry of carbonatites and related rocks from two carbonatite complexes, south Nyanza, Kenya. Lithos 7, 53-63.

Bailey, D.K. (1993) Carbonate magmas. J. Geol. Soc. 150, 637-651.

Bedard, L.P. \& Chown, E.H. (1992) The Dolodau Dykes, Canada: an example of an Archean carbonatite. Mineral. and Petrol. 46, 109-121.

Bowden, P. (1962) Trace elements in Tanganyika carbonatites. Nature 196, 570.

Bowden, P. (1968) Trace elements in carbonatites and limestones. Nature 219, 716.

Buddington, A.F. \& Lindsley, D.H. (1964) Iron-titanium oxide minerals and synthetic equivalents.

J. Petrol. 5, 310-357.

Burt, D.M. (1989) Compositional and phase relations among rare earth element minerals. Pp. 259-307 in B.R. Lipin \& G.A. McKay (eds) Geochemistry and mineralogy of rare earth elements. Rev. in Miner. 21. Mineral. Soc. Amer.

Cullers, R.L. \& Graf, J.L. (1984) Rare earth elements in igneous rocks of the continental crust: predominantly basic and ultrabasic rocks. Pp. 237-274 in P. Henderson (ed.) Developments in Geochemistry, Vol. 2: rare earth elements geochemistry. Elsevier, Amsterdam.

Deines, P. (1989) Stable isotope variations in carbonatites. Pp. 301-359 in K. Bell (ed.), Carbonatites: Genesis and Evolution. Unwin Hyman, London,

Eby, G.N. (1975) Abundance and distribution of the rare-earth elements and yttrium in the rocks and minerals of the Oka carbonatite complex, Quebec. Geochim. Cosmochim. Acta 39, 597-620.

Epstein, S., Graf, D.L. \& Degens, E.T. (1964) Oxygen isotope studies on the origin of dolomites. Pp. 169-180 in H. Craig, S.L. Miller and G.J. Wasserburg (eds) Isotopic and Cosmic Chemistry. North-Holland Publishing Co., Amsterdam.

Gaspar, J.C. (1992) Titanian clinohumite in the carbonatites of the Jacupiranga complex, Brazil: mineral chemistry and comparison with titanian clinohumite from other environments. Am. Mineral. $77,168-178$.

Gaspar, J.C. \& Wyllie, P.J. (1987) The phlogopites from the Jacupiranga carbonatite intrusions. Mineral. and Petrol. 36, 121-134.

Gittins, J. (1989) The origin and evolution of carbonatite magmas. Pp. 580-600 in K. Bell (ed.)
Carbonatites: genesis and evolution. Unwin Hyman, London.

Gregory, R.T. \& Criss, R.E. (1986) Isotopic exchange in open and closed systems. Pp. 91-127 in J.W. Valley, H.P. Taylor, Jr. and J.R. O'Neil (eds) Stable Isotopes in High Temperature Geological Processes. Rev. in Mineral., 16, Mineral. Soc. America.

Haggerty, S.E. (1991) Oxide textures - a mini-atlas. Pp. 129-219 in D.H. Lindsley (ed.) Oxide minerals: petrologic and magnetic significance. Rev. in Miner. 25. Mineral. Soc. Amer.

Heinrich, E.Wm. (1966) The geology of carbonatites. Rand McNally \& Company, Chigago. 555 pp.

Hogarth, D.D. (1989) Pyrochlore, apatite and amphibole: distinctive minerals in carbonatite. Pp. 105148 in K. Bell (ed.) Carbonatites: genesis and evolution. Unwin Hyman, London.

Hogarth, D.D., Rushforth, P. \& McCorkell, R.H. (1988) The Blackburn carbonatites, near Ottawa, Ontario: dykes with fluidized emplacement. Can. Mineral. 26, 377-390.

Hogarth, D.D., Hartree, R., Loop, J. \& Solberg, T.N. (1985) Rare-earth element minerals in four carbonatites near Gatineau, Quebec. Am. Mineral. 70: 1135-1142.

Honkamo, M. (1979) Kallioperäkartta, lehti 3541, Rytinki. Suomen geologinen kartta 1:100 000.

Jenkin, G.R.T., Linclater, C. \& Fallick, A.E. (1991) Modeling of mineral ${ }^{18} \mathrm{O}$ values in an igneous aureole: Closed-system model predicts apparent open-system ${ }^{18} \mathrm{O}$ values. Geology, 19: 1185-1188.

Kapustin, Yu.L. (1966) Geochemistry of rare-earth elements in carbonatites. Geochemistry International 3, 1054-1065.

Kapustin, Yu.L. (1980) Mineralogy of carbonatites, D.K. Biswas (English translation). Amerind, New Delhi. 259 pp.

Karhu, J.A. (1993) Paleoproterozoic evolution of the carbon isotope ratios of sedimentary carbonates in the Fennoscandian Shield. Geol. Surv. Finl. Bull., $371,1-87$.

Kärki, A., Laajoki, K., \& Luukas, J. (1993) Major early Proterozoic shear zones of Central Fennoscandian Shield. Precambr. Res. 64, 207-223.

Kocman, V. \& Rucklidge, J. (1973) The crystal structure of a titaniferous clinohumite. Can. Min. 12,39-45.

Kresten, P., Printzlau, I., Rex, D., Vartiainen, H. \& Woolley, A. (1977) New ages of carbonatitic and alkaline ultramafic rocks from Sweden and Finland. Geol. Fören. Stockholm Förh. vol. 99, 62-65. 
Kubicki, S. \& Ryka, W. (1987) Carbonatites of the alkaline-ultramafic intrusion of Tajno / Precambrian basement of Poland. Pp. 11-21 in L. Kopecky (ed.) Proceedings of the first seminar on carbonatites and alkaline rocks of the Bohemian massif and ambient regions, Prague, Czechoslovakia, May 23, 1984. Prague, the Geological Survey.

Kyser, T.K. (1987) Equilibrium fractionation factors for stable isotopes. In: T.K. Kyser (ed.) Stable Isotope Geochemistry of Low Temperature Processes. Short Course Handbook, 13, Mineral. Assoc. Canada, pp. 1-84.

Laajoki, K. (1991) Stratigraphy of the northern end of the early Proterozoic (Karelian) Kainuu Schist Belt and associated gneiss complexes, Finland. Geol. Surv. Finland, Bull. 358, 105 pp.

Le Bas, M.J. (1977) Carbonatite-nephelinite volcanism. John Wiley \& Sons, London. 347 pp.

Leake, B.E., Woolley, A.R., Arps, C.E.S., Birch, W.D., Gilbert, M.C., Grice, K.D., Hawthorne, F.C., Kato, A., Kisch, H.J., Krivovichev, V. G., Linthout, K., Laird, J., Mandarino, J.A., Maresch W.V., Nickel, E.H., Rock, N.M.S., Schumacher, J.C., Smith, D.C., Stephenson, N.C.N., Ungaretti, L., Whittater \& E.J.W. Youzhi, G. (1997) Nomenclature of amphiboles. Report of the Subcommitte on Amphiboles of the International Mineralogical Association, Commission on New Minerals and Mineral Names. Can. Mineral. 35, 219-246.

Loubet, M., Bernat, M., Javoy, M. \& Allegre, C.J. (1972) Rare earth contents in carbonatites. Earth Plan. Sci. Lett. 14, 226-232.

Mariano, A.N. (1989a) Nature of economic mineralization in carbonatites and related rocks. Pp. 149176 in K. Bell (ed.) Carbonatites: genesis and evolution. Unwin Hyman, London.

Mariano, A.N. (1989b) Economic geology of rare earth minerals. Pp. 309-334 in B.R. Lipin \& G.A. McKay (eds) Geochemistry and mineralogy of rare earth elements. Rev. in Miner. 21. Mineral. Soc. Amer.

Mitchell, R.H. (1979) The alleged kimberlite-carbonatite relationship: additional contrary mineralogical evidence. Am. J. Sci. 279, 570-589.

Mitchell, R.H. (1986) Kimberlites: mineralogy, geochemistry and petrology. Plenum Press, New York. $442 \mathrm{pp}$.

Mitchell, R.H. \& Brunfelt, A.O. (1975) Rare earth element geochemistry of the Fen alkaline complex, Norway. Contrib. Mineral. Petrol. 52, 247-259.

Möller, P. (1989a) REE(Y), Nb, and Ta enrichment in pegmatites and carbonatite-alkalic rock comp- lexes. Pp. 103-144 in P. Möller, P. Cerny \& F. Saupé (eds) Lanthanides, tantalum and niobium. Mineralogy, geochemistry, characteristics of primary ore deposits, prospecting, processing and applications. Proceedings of a workshop in Berlin, November 1986. Springer-Verlag, Berlin.

Möller, P. (1989b) Rare earth mineral deposits and their industrial importance. Pp. 171-188 in P. Möller, P. Cerny ja F. Saupé (eds) Lanthanides, tantalum and niobium. Mineralogy, geochemistry, characteristic of primary ore deposits, prospecting, processing and applications. Proceedings of a workshop in Berlin, November 1986. SpringerVerlag, Berlin.

Möller, P., Morteani, G. \& Schley, F. (1980) Discussion of REE distribution patterns of carbonatites and alkalic rocks. Lithos 13, 171-179.

Morteani, G. (1989) Prospection for niobium-rich alkaline rocks. Pp. 311-320 in P. Möller, P. Cerny ja F. Saupé (eds) Lanthanides, tantalum and niobium. Mineralogy, geochemistry, characteristics of primary ore deposits, prospecting, processing and applications. Proceedings of a workshop in Berlin, November 1986. Springer-Verlag, Berlin.

Nelson, D.R., Chivas, A.R., Chappell, B.W. \& McCulloch, M.T. (1988) Geochemical and isotopic systematics in carbonatites and implications for the evolution of ocean-island sources. Geochim. Cosmochim. Acta 52, 1-17.

Northrop, D.A. \& Clayton, R.N. (1966) Oxygen isotope fractionations in systems containing dolomite. J. Geol., 74, 174-196.

Nykänen, J. (1993) Pudasjärven Kortejärven ja Posion Laivajoen proterotsooisten karbonatiittien geologia, mineralogia ja geokemia. Geology, mineralogy and geochemistry of the Proterozoic Kortejärvi and Laivajoki carbonatites in Pudasjärvi and Posio. Unpubl. M. Sc. Thesis, Univ. of Oulu, Dept. of Geology, 60 pp.

Pell, J. \& Höy, T. (1989) Carbonatites in a continental margin environment - The Canadian Cordillera. Pp. 200-220 in K. Bell (ed.) Carbonatites: genesis and evolution. Unwin Hyman, London.

Prins, P. (1972) Composition of magnetite from carbonatites. Lithos 5, 227-240.

Rock, N.M.S. (1987) A Fortran program for tabulating and naming amphibole analyses according to the International Mineralogical Association scheme. Mineral. and Petrol. 37, 79-88.

Samoilov, V.S. (1991) The main geochemical features of carbonatites. J. Geochem. Explor. 40, 251-262. 
Sheppard, S.M.F. \& Schwarcz, H.P. (1970) Fractionation of carbon and oxygen isotopes and magnesium between coexisting metamorphic calcite and dolomite. Contrib. Mineral. and Petrol. 26, 161-198.

Silvennoinen, A. (1991) Kuusamon ja Rukatunturin kartta-alueiden kallioperä. Summary: Pre-Quaternary rocks of the Kuusamo and Rukatunturi mapsheet areas. Geological map of Finland 1:100 000. Explanation to the Maps of Pre-Quaternary rocks, 4524+4542 Kuusamo and 4613 Rukatunturi. 63 pp.

Sokolov, S.V. (1985) Carbonates in ultramafite, alkali-rock, and carbonatite intrusions. Geochemistry International 22, 150-166.

Spear, F.S. \& Kimball, K.L. (1984) Recamp - a Fortran IV program for estimating $\mathrm{Fe}^{3+}$ contents in amphiboles. Computers \& Geosciences 10, 317-325.

Vartiainen, H. \& Woolley, A.R. (1974) The age of the Sokli carbonatite, Finland, and some relationships of the North Atlantic alkaline igneous province. Bull. Geol. Soc. Finland 46, 81-91.

Wall, F. \& Mariano, A.N. (1996) Rare earth minerals in carbonatites: a discussion centered on the Kangankunde Carbonatite, Malawi. Pp. 193-225 in A.P. Jones, F. Wall and C.T.Williams (eds) Rare Earth Minerals. Chemistry, Origin and Ore Deposits. The mineralogical Society Series 7. Chapman and Hall, London.

Woolley, A.R. (1989) The spatial and temporal distribution of carbonatites. Pp. 15-37 in K. Bell (ed.) Carbonatites: genesis and evolution. Unwin Hyman, London.

Woolley, A.R. \& Buckley, H.A. (1993) Magnesite-siderite series carbonates in the Nkombwa and Newania carbobnatite complexes. S. Afr. J. Geol. 96, 126-130.

Woolley, A.R. \& Kempe, D.R.C. (1989) Carbonatites: nomenclature, average chemical compositions, and element distribution. Pp. 1-14 in K. Bell (ed.) Carbonatites: genesis and evolution. Unwin $\mathrm{Hy}$ man, London. 